Quasielastic and inelastic neutron scattering investigation of fragile-to-strong crossover in deeply supercooled water confined in nanoporous silica matrices

This article has been downloaded from IOPscience. Please scroll down to see the full text article.

2006 J. Phys.: Condens. Matter 18 S2261

(http://iopscience.iop.org/0953-8984/18/36/S03)

The Table of Contents and more related content is available

Download details:

IP Address: 140.112.113.225

The article was downloaded on 11/08/2009 at 10:02

Please note that terms and conditions apply. 


\title{
Quasielastic and inelastic neutron scattering investigation of fragile-to-strong crossover in deeply supercooled water confined in nanoporous silica matrices
}

\author{
Li Liu ${ }^{1}$, Sow-Hsin Chen ${ }^{1,6}$, Antonio Faraone ${ }^{2,3}$, Chun-Wan Yen ${ }^{4}$, \\ Chung-Yuan $\mathrm{Mou}^{4}$, Alexander I Kolesnikov ${ }^{5}$, Eugene Mamontov ${ }^{2,3}$ and \\ Juscelino Leao ${ }^{2,3}$ \\ ${ }^{1}$ Department of Nuclear Science and Engineering, Massachusetts Institute of Technology, \\ 77 Massachusetts Avenue, Cambridge, MA 02139, USA \\ 2 NIST Center for Neutron Research, NIST, Gaithersburg, MD 20899, USA \\ 3 Department of Materials Science and Engineering, University of Maryland, College Park, MD \\ 20742, USA \\ ${ }^{4}$ Department of Chemistry, National Taiwan University, Taipei, Taiwan \\ ${ }^{5}$ Intense Pulsed Neutron Source Division, Argonne National Laboratory, Argonne, IL 60439, USA \\ E-mail: sowhsin@mit.edu
}

Received 23 January 2006

Published 24 August 2006

Online at stacks.iop.org/JPhysCM/18/S2261

\begin{abstract}
We investigated, using quasi-elastic and inelastic neutron scattering, the slow single-particle dynamics of water confined in laboratory synthesized nanoporous silica matrices, MCM-41-S, with pore diameters ranging from 10 to $18 \AA$. Inside the pores of these matrices, the freezing process of water is strongly inhibited down to $160 \mathrm{~K}$. We analysed the quasi-elastic part of the neutron scattering spectra with a relaxing-cage model and determined the temperature and pressure dependence of the $Q$-dependent translational relaxation time and its stretch exponent $\beta$ for the time dependence of the self-intermediate scattering function. The calculated $Q$-independent average translational relaxation time shows a fragile-to-strong (FS) dynamic crossover for pressures lower than 1600 bar. Above this pressure, it is no longer possible to discern the characteristic feature of the FS crossover. Identification of this end point with the predicted second low-temperature critical point of water is discussed. A subsequent inelastic neutron scattering investigation of the librational band of water indicates that this FS dynamic crossover is associated with a structural change of the hydrogen-bond cage surrounding a typical water molecule from a denser liquid-like configuration to a less-dense ice-like open structure.
\end{abstract}

(Some figures in this article are in colour only in the electronic version)

6 Author to whom any correspondence should be addressed. 


\section{Introduction}

Glass is an amorphous solid form of matter that results when a fluid is supercooled or compressed in such a way that it bypasses crystallization. Many types of materials are capable of glass formation, such as molecular liquids, polymers, metal alloys and molten salts. Given such diversity of materials, a general scheme, by which different glass-forming materials can be systematically classified according to their relaxational behaviours, is useful. One such scheme is the classification of glass-formers according to their 'fragility'. Fragility measures the rate with which transport properties of a liquid, such as structural relaxation time, viscosity or the inverse self-diffusion constant, change as the glassy state is approached from the liquid side by lowering temperature.

By convention, the glass transition temperature $T_{\mathrm{g}}$ is where the viscosity $\eta$ reaches a value of $10^{12} \mathrm{~Pa}$ s or the structural relaxation time $\tau_{T}$ reaches an order of $100 \mathrm{~s}$. The approach to this large $\eta$ or $\tau_{T}$, however, differs from one liquid to another. When displayed in an Arrhenius plot of $\log \eta\left(\right.$ or $\left.\log \tau_{T}\right)$ versus inverse temperature $1 / T$, some liquids (such as silica) show a steady, linear increase, while others display a much steeper dependence on $1 / T$ (such as $o$-terphenyl). The former are called 'strong' liquids, and the latter, 'fragile' liquids. Thus, the glassy liquid is called 'fragile' when its viscosity or relaxation time varies according to super-Arrhenius law, such as the Vogel-Fulcher-Tammann (VFT) law:

$$
\tau_{T}=\tau_{1} \mathrm{e}^{D T_{0} /\left(T-T_{0}\right)}
$$

where $T_{0}$ is the temperature of apparent divergence of the relaxation time (or sometime called Kauzmann temperature); the magnitude of $D$ gives the degree of fragility; and $\tau_{1}$ is a prefactor related to microscopic vibrational relaxation time inside the cage forming by neighbours in the liquid state. The liquid is called 'strong' when the viscosity or relaxation time obeys Arrhenius law:

$$
\tau_{T}=\tau_{1} \mathrm{e}^{E_{\mathrm{A}} / R T}
$$

where $E_{\mathrm{A}}$ is the energy barrier for the relaxation process and $R$ the gas constant [1].

For water, which is a fragile liquid at room temperature and at moderately supercooled temperatures, Angell and co-workers [2] proposed that a 'fragile-to-strong' (FS) transition would occur at around $228 \mathrm{~K}$ at ambient pressure, based on a thermodynamic argument. But supercooled water nucleates into hexagonal ice at and below $T_{\mathrm{H}}=235 \mathrm{~K}$, so this transition lies in an inaccessible region of temperatures and it has not been observed directly so far [3]. By containing water in the small cylindrical pores (pore size $\leqslant 18 \AA$ ) of MCM-41-S, we were able to circumvent the homogenous nucleation process and supercool water down to $160 \mathrm{~K}$.

The interest of the scientific community in the properties of water confined in nanoporous matrices at supercooled temperatures has a twofold basis. On the one hand, confining water in nanometric cavities allows us to study water in deeply supercooled states. This temperature range is of fundamental importance to the science of water. At ambient pressure, bulk liquid water shows an anomalous increase of thermodynamic quantities and an apparent divergent behaviour of transport properties, on approaching the singular temperature $T_{\mathrm{c}}=228 \mathrm{~K}$ [4], which lies below $T_{\mathrm{H}}$. Thus, there is a lack of experimental data in this temperature range, which hampers the verification or the rejection of the different physical pictures proposed to explain the origin of the anomalous behaviour of water. On the other hand, in many real life systems water is not in its bulk form but is located near surfaces or contained in small cavities. This is the case, for example, for water in rocks, in polymer gels, and in biological membranes [5]. In addition, the properties of water in porous silica glasses, such as Vycor, and silica gel, are relevant in catalytic and separation processes. The above two lines of interest are 
obviously closely related, as the clarification of the fundamental properties of water is likely to be the key to understanding the behaviour of many real systems. For example, in the case of enzymatic activity of protein, it has been found that the onset of protein activities is strongly correlated to the onset of orientational fluctuations that initiate structural rearrangements within the transient $\mathrm{H}$-bond water network surrounding the protein $[6,7]$. Therefore, the study of the dynamics of water confined in nanopores as a function of temperature and pressure is relevant in understanding important effects in systems of interest in biology, chemistry, and geophysics $[8,9]$.

Due to the facts mentioned above, both the structure and dynamics of water in confined geometries have been widely studied using molecular dynamics (MD) simulations and different experimental techniques. MD simulations of the extended simple point charge (SPC/E) model of bulk water have furnished relevant results for understanding the dynamics of supercooled water [10-14]. From the analysis of these results, the relaxing-cage models (RCMs) for the translational and rotational dynamics of water at supercooled temperatures have been developed [10, 11]. In addition to this, MD simulations of SPC/E water confined in silica nanopores have been carried out as well $[15,16]$. The interaction between the hydrophilic surface and the water molecules has noticeable effects on the structure of the first and second layer of water near the pore surface [17]. As far as the dynamics is concerned, when confined near hydrophilic surfaces, water molecules are in the glassy state. In general, the water molecules show a dynamics similar to that of supercooled water $[15,16]$ at a lower equivalent temperature of some $30^{\circ}[6,18]$.

Experimentally, the structural properties of water at supercooled temperatures [19] and in confinement [20] have been studied extensively using x-ray and neutron diffraction. On the other hand, the relaxational dynamics of water confined in mesoporous matrices have been studied using dielectric spectroscopy [3, 21-25] and different nuclear magnetic resonance (NMR) techniques [26-29].

Quasielastic neutron scattering (QENS) and inelastic neutron scattering (INS) techniques offer many advantages for the study of the single particle dynamics of water. The main reason is that the total scattering cross section of hydrogen is much larger than that of atoms in silica, composed of oxygen and silicon. Furthermore, the neutron scattering of hydrogen atoms is mostly incoherent so that QENS spectra reflect, essentially, the self-dynamics of the hydrogen atoms in water. By combining this dominant cross section of hydrogen atoms with the use of spectrometers having different energy resolutions, we can study the self-dynamics of water in a wide range of time-scale, encompassing picosecond to tens of nanoseconds. In addition, by investigating different $Q$ values ( $Q$ being the magnitude of the exchanged wavevector) in the range from $0.2 \AA^{-1} \leqslant Q \leqslant 2.00 \AA^{-1}$, the spatial characteristics of water dynamics can be investigated at a molecular level.

The experimentally determined double differential scattering cross section is directly related to the self-dynamic structure factor, $S_{\mathrm{H}}(Q, \omega)$, which is the Fourier transform of the self-intermediate scattering function (ISF), $F_{\mathrm{H}}(Q, t)$, of the hydrogen atom in a water molecule. This connection facilitates the interpretation of the scattering data and allows a direct comparison with theory and with the data from MD simulations.

This paper presents a systematic investigation of temperature and pressure dependences of dynamics of water inside nanoporous silica materials. The temperature range covers from room temperature down to deeply supercooled states, and the pressure ranges from ambient to 2400 bar. QENS data collected are analysed according to a single consistent model RCM, which is valid for bulk as well as confined water as was shown by MD simulation [10-16]. 


\section{Preparation and characterization of the samples}

Micellar templated mesoporous silica matrices MCM-41-S have 1D cylindrical pores arranged in 2D hexagonal arrays. Similar to synthesizing MCM-48-S [30], to make the mesoporous materials together with short surfactant $\left(\mathrm{C}_{12} \mathrm{TMAB}\right)$, we employed small quaternary ammonium ions, TEAOH, to separately develop a zeolitic nanocluster as the silica precursor. In this way, we will get MCM-41-S with smaller pore sizes and stronger silica walls than traditional ways $[31,32]^{7}$. Initially, sodium aluminate, sodium hydroxide, and tetraethylammonium hydroxide ( $20 \mathrm{wt} \%$ aqueous solution) were mixed in a vessel. Then the fumed silica was added into the above mixture and the whole system was stirred for $4 \mathrm{~h}$. The solution was transferred to autoclaves and heated at $100^{\circ} \mathrm{C}$ for $18 \mathrm{~h}$, yielding zeolite precursors. $A$ mixture of $\mathrm{C}_{12} \mathrm{TMAB}$ or $\mathrm{C}_{10} \mathrm{TMAB}$ and deionized water was added into the zeolite precursors. The resulting mixture was transferred to autoclaves, and again heated at $120-150{ }^{\circ} \mathrm{C}$ in an oven. After $18-48 \mathrm{~h}$ of crystallization, the solid product was filtered, washed with water, and dried at $60^{\circ} \mathrm{C}$ in air for $2 \mathrm{~h}$. Calcination of the sample was carried out at $580^{\circ} \mathrm{C}$ for $6 \mathrm{~h}$ in air to remove the templates. (The molar ratio of reactants is $\left.\mathrm{NaAlO}_{2}: \mathrm{SiO}_{2}: \mathrm{NaOH}: \mathrm{TEAOH}: \mathrm{C}_{12} \mathrm{TMAB}: \mathrm{H}_{2} \mathrm{O}=1: 37-60: 1.5-9: 11-22: 18.3: 3000-3500\right)$.

The synthesized samples were characterized using x-ray powder diffraction (XRD), nitrogen adsorption-desorption, and differential scanning calorimetry (DSC).

XRD were acquired with a Scintag X1 diffractometer, using $\mathrm{Cu} \mathrm{K} \alpha(40 \mathrm{kV}, 30 \mathrm{~mA}$, $\lambda=0.154 \mathrm{~nm})$. The XRD patterns of the samples show that the MCM-41-S had hexagonal $(P 6 \mathrm{~mm})$ symmetry, as shown in figure 1. All the samples exhibited high hydrothermal stability.

The melting/freezing behaviour of water in the samples (fully hydrated) was checked by DSC measurements (TA Instrument 5100 control system and a LT-Modulate DSC 2920) (figure 2). According to the Gibbs-Thomson equation, the melting point of a small crystal is proportional to the crystal size, which, in this case, is equal to the pore size of the material. Thus, one expects that the liquid state of water would persist to very low temperature if the pore size can be decreased further. In figure 2, the DSC curve shows the melting points which are specified by the temperatures at the positions of the inverted peaks. For samples with pore size $\geqslant 19 \AA$, we do see a small peak near $0{ }^{\circ} \mathrm{C}$ which is due to the water outside the nanochannel(unconfined). For samples having pore sizes $\leqslant 18 \AA$, we do not see any abrupt melting transition near $0{ }^{\circ} \mathrm{C}$, indicating that there is no water residing outside the channel. But, we seem to see a gradual change of enthalpy from -100 to $-50^{\circ} \mathrm{C}$. This could be due to some second-order transition or glass transition.

In the region of small angles $(2 \theta=1.5-8)$, the Brunaumer-Emmet-Teller (BET) surface area, pore size and pore volume were determined using nitrogen adsorption-desorption at $77 \mathrm{~K}$ (Micromeritics ASAP 2010 sorptometer at $77 \mathrm{~K}$ ) (figure 3). The obtained values are reported in table 1. On all the curves, one can see a sharp rise of the adsorption at vapour pressure near zero which can give the surface area by fitting to a BET adsorption isotherm. The sharp rise at intermediate pressure is due to capillary condensation into the mesopore. The smaller the pore size is, the lower the pressure at which this condensation would occur. And the sharper this capillary rise is, the narrower pore size distribution one has. There is a gradual decrease of pore size from top to bottom curves. The pore sizes are determined by the Barrett-Joyner-Halenda (BJH) method, which is based on equilibrium capillary thermodynamics.

\footnotetext{
7 The MCM-41-S materials are able to withstand prolonged ( $>1$ month) exposure to water at room temperature without structural decay. The pore size is determined by capillary condensation in the standard Barrett-Joyner-Halenda $(\mathrm{BJH})$ method in a nitrogen adsorption experiment (at $77 \mathrm{~K}$ ). Because of the uncertainty in estimating the thickness of the surface immobile layer, the pore size is a nominal estimation.
} 


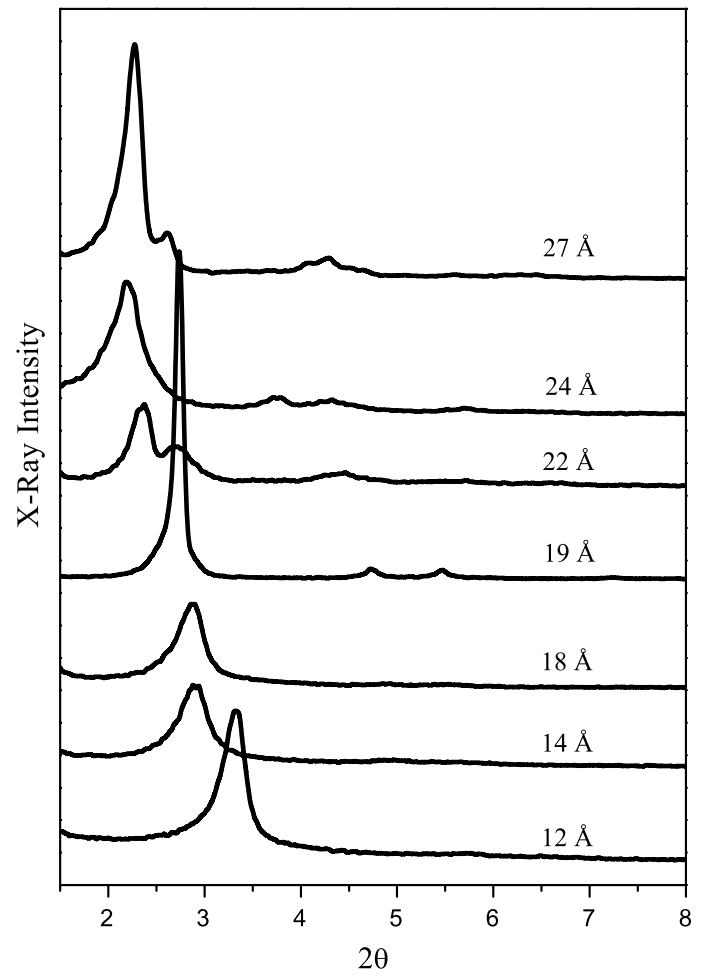

Figure 1. This figure shows XRD patterns of MCM-41-S samples with different pore sizes taken at room temperature and ambient pressure. The sharp XRD peaks show that the samples are wellordered. The (100) peak (the highest peak) is related to the $d$-spacing according to the rule: $2 d$ $\sin (\theta)=\lambda$, where $\lambda=1.54 \AA$ and $\theta$ is in degrees. Since the samples do not show a peak at the high angle region, it means that we have just one phase (pure phase). The number above each curve indicates the average pore diameter of that sample.

Table 1. Parameters characterizing the structural properties of the investigated samples.

\begin{tabular}{lllllll}
\hline & Samples & $\begin{array}{l}\text { Surface area } \\
\left(\mathrm{m}^{2} \mathrm{~g}^{-1}\right)\end{array}$ & $\begin{array}{l}\text { Pore size } \\
(\AA)\end{array}$ & $\begin{array}{l}\text { Pore volume } \\
\left(\mathrm{cm}^{3} \mathrm{~g}^{-1}\right)\end{array}$ & $\begin{array}{l}\text { Hydration level } \\
(\mathrm{wt} \%)\end{array}$ & $\begin{array}{l}\text { Elastic component } \\
p\end{array}$ \\
\hline A & MCM-27 & 1312 & 27.0 & 0.97 & 48 & 0.0 \\
B & MCM-24 & 1358 & 24.0 & 1.38 & 50 & 0.0 \\
C & MCM-22 & 1156 & 22.0 & 1.08 & 51 & 0.0 \\
D & MCM-19 & 1170 & 19.0 & 0.76 & 49 & 0.0 \\
E & MCM-18 & 1384 & 18.0 & 0.67 & 55 & 0.069 \\
F & MCM-14 & 726 & 14.0 & 0.41 & 50 & 0.079 \\
G & MCM-12 & 1018 & 12.0 & 0.65 & 48 & 0.106 \\
H & MCM-10 & 875 & 10.0 & 0.49 & 40 & 0.108 \\
\hline
\end{tabular}

\section{QENS and INS experiments}

High-resolution QENS experiments were used to detect the dynamic transition in water by directly measuring the average translational relaxation time, $\left\langle\tau_{T}\right\rangle$, of water molecules in the temperature range from $325 \mathrm{~K}$ down to deeply supercooled temperature, $160 \mathrm{~K}$ under pressure. Two QENS spectrometers in NIST Center for Neutron Research (NIST NCNR) were used: a disc-chopper time-of-flight spectrometer (DCS) and a high-flux backscattering spectrometer 


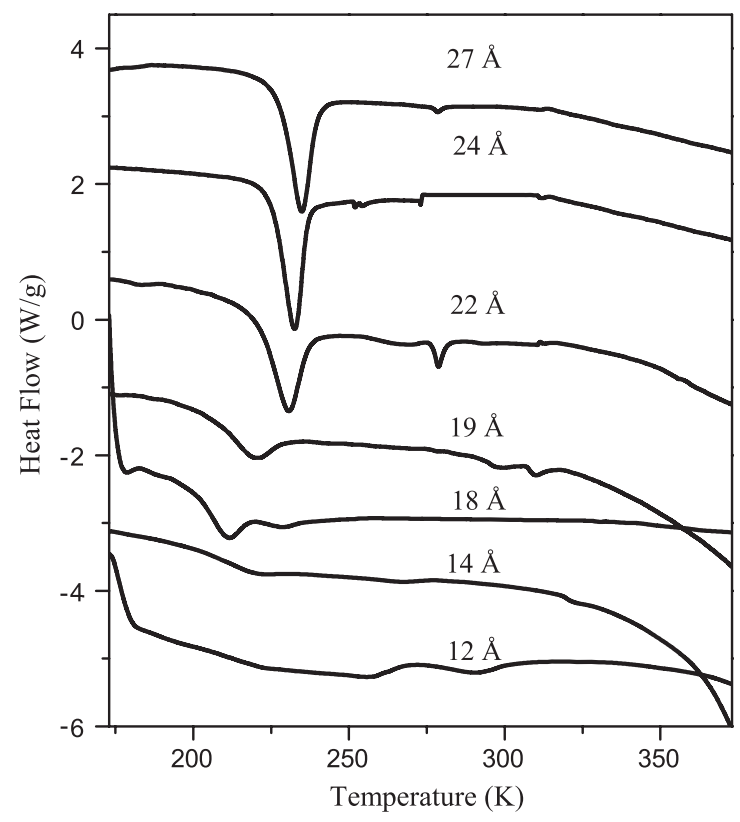

Figure 2. DSC curves of water inside MCM-41-S samples having different pore sizes (indicated by the numbers above the curves). The sharp negative-going peaks signal the freezing temperatures. It is noted that the samples with pore size $\leqslant 18 \AA$ do not show an obvious freezing peak.

(HFBS). For the DCS [33], the incident neutron wavelength was $9.0 \AA$ and the Gaussian energy resolution function had a full width at half maximum (FWHM) of $\sim 20 \mu \mathrm{eV}$ and a dynamic range of $\pm 0.5 \mathrm{meV}$. For the HFBS [34], we chose the high-resolution configuration, corresponding to a resolution function with FWHM of $\sim 0.8 \mu \mathrm{eV}$ and an energy window of $\pm 11 \mu \mathrm{eV}$. Combination of results from the above two spectrometers enables us to cover the relaxation time range from $1 \mathrm{ps}$ at high temperatures to $10 \mathrm{~ns}$ at the lowest temperature. The resulting range of elastic wavevector $Q$ was from 0.25 to $1.75 \AA^{-1}$, and from 0.07 to $1.30 \AA^{-1}$, in the case of the DCS and HFBS, respectively. The spectra were corrected for scattering from the sample holder, standardized using results from a vanadium run, and converted to the differential scattering cross section using standard routines available at NIST.

INS measurements of the librational band of water were carried out at the high-resolution medium-energy chopper spectrometer (HRMECS) of the Intense Pulsed Neutron Source at Argonne National Laboratory (IPNS ANL), with $150 \mathrm{meV}$ incident energy and an energy window 0-130 meV [35].

For both QENS and INS, we used a high-pressure system, including a specially designed aluminium pressure cell, to apply pressure to the samples. Helium gas was used as the pressuretransmitting medium. The gas was supplied through a small capillary tube from the pressure system to the hydrated sample. The experiment at each pressure was done with a series of temperatures, covering both below and above the crossover temperature. Altogether, over 1100 QENS spectra and over 30 INS spectra were collected, spanning ten pressures: ambient, 100, 200, 400, 800, 1200, 1400, 1600, 2000, and 2400 bars.

\section{Relaxing-cage model (RCM) for the incoherent intermediate scattering function}

During the past several years, we have developed the relaxing-cage model (RCM) for the description of translational and rotational dynamics of water at supercooled temperatures. 


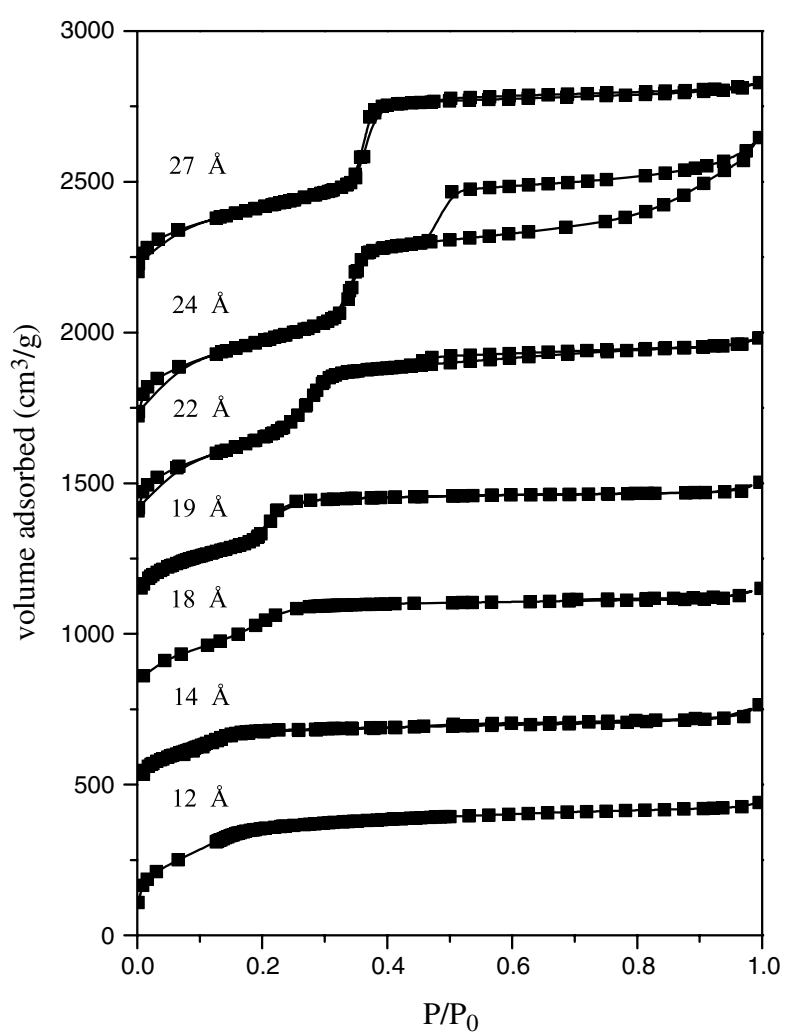

Figure 3. The nitrogen adsorption-desorption at $77 \mathrm{~K}$ curve of MCM samples with different pore sizes. One can see a sharp rise of the adsorption at vapour pressure near zero which can give surface area by fitting to a BET adsorption isotherm. The sharp rise at intermediate pressure is due to capillary condensation into the mesopore. The sharper this capillary rise is, the narrower pore size distribution one has. The pore sizes are determined by the Barrett-Joyner-Halenda (BJH) method, which is based on equilibrium capillary thermodynamics. The BET diagram of the $24 \AA$ sample shows a hysteresis loop in the desorption branch because the sample contains extensive void defects which display this type of hysteresis loop in the nitrogen desorption curve.

These models have been tested with MD simulations of SPC/E water, and have been used to analyse QENS data [36-40].

The experimentally determined double differential scattering cross section $\mathrm{d}^{2} \sigma / \mathrm{d} \Omega \mathrm{d} \omega$ is proportional to the self-dynamic structure factor of hydrogen atoms $S_{\mathrm{H}}(Q, \omega)$ through the following relation:

$$
\frac{\mathrm{d}^{2} \sigma_{\mathrm{H}}}{\mathrm{d} \Omega \mathrm{d} \omega}=N \frac{\sigma_{\mathrm{H}}}{4 \pi} \frac{k_{f}}{k_{\mathrm{i}}} S_{\mathrm{H}}(Q, \omega),
$$

where $E=\hbar \omega$, the energy exchanged by the neutron to the sample; $\mathrm{d} \Omega$, the solid angle into which the neutron is scattered; $N$, the number of scattering centres in the scattering volume; $k_{f}$, the scattered wavevector; $k_{\mathrm{i}}$, the incident wavevector; and $\sigma_{\mathrm{H}}$, the incoherent scattering cross section of a hydrogen atom. The dynamic structure factor, $S_{\mathrm{H}}(Q, \omega)$, is the Fourier transform of the self-intermediate scattering function (ISF) of hydrogen atoms, $F_{\mathrm{H}}(Q, t)=\exp \{\mathrm{i} \vec{Q} \cdot[\vec{r}(t)-\vec{r}(0)]\}$, according to the equation

$$
S_{\mathrm{H}}(Q, \omega)=\frac{1}{2 \pi} \int_{-\infty}^{\infty} \mathrm{d} t \mathrm{e}^{-\mathrm{i} \omega t} F_{\mathrm{H}}(Q, t) .
$$


$F_{\mathrm{H}}(Q, t)$ is, then, the primary quantity of theoretical interest related to experiments. It can be calculated by a model, such as the RCM, and a molecular dynamics simulation of a phenomenological model of water.

For QENS data analysis purposes, the full dynamics of bulk or confined water should include both the translational and the rotational motion of a rigid water molecule. Given the fact that in the process of QENS data analysis, we only focus our attention on the ISF with $Q \leqslant 1.1 \AA^{-1}$, we can safely neglect the contribution of rotational motion to the total dynamics [41], which means that $F_{\mathrm{H}}(Q, t) \approx F_{T}(Q, t)$.

On lowering the temperature below the freezing point, around a given water molecule there is a tendency to form a hydrogen-bonded, tetrahedrally coordinated first and second neighbour shell (cage). At short times, less than $0.05 \mathrm{ps}$, the centre of mass of a water molecule performs vibrations inside the cage. At long times, longer than $1.0 \mathrm{ps}$, the cage eventually relaxes and the trapped particle can migrate through the rearrangement of a large number of particles surrounding it. Therefore, there is a strong coupling between the single particle motion and the density fluctuations of the fluid. This physical picture is the so-called relaxing-cage model.

The RCM assumes that the translational short-time dynamics of the trapped water molecule can be treated approximately as the motion of the centre of mass in an isotropic harmonic potential well, provided by the mean field of its neighbours. We can, then, write the shorttime part of the translational ISF in a Gaussian approximation, connecting it to the velocity auto-correlation function, $\left\langle\vec{v}_{\mathrm{CM}}(t) \cdot \vec{v}_{\mathrm{CM}}(0)\right\rangle$, in the following way:

$F_{T}^{s}(Q, t)=\exp \left\{-Q^{2} \frac{\left\langle r_{\mathrm{CM}}^{2}(t)\right\rangle}{2}\right\}=\exp \left\{-Q^{2}\left[\int_{0}^{t}(t-\tau)\left\langle\vec{v}_{\mathrm{CM}}(0) \cdot \vec{v}_{\mathrm{CM}}(\tau)\right\rangle \mathrm{d} \tau\right]\right\}$.

Since the translational density of states, $Z_{T}(\omega)$, is the Fourier transform of the normalized centre of mass velocity auto-correlation function, one can express the mean squared deviation, $\left\langle r_{\mathrm{CM}}^{2}(t)\right\rangle$, as follows:

$$
\left\langle r_{\mathrm{CM}}^{2}(t)\right\rangle=\frac{2}{3}\left\langle v_{\mathrm{CM}}^{2}\right\rangle \int_{0}^{\infty} \mathrm{d} \omega \frac{Z_{T}(\omega)}{\omega^{2}}(1-\cos \omega t),
$$

where $\left\langle v_{\mathrm{CM}}^{2}\right\rangle=\left\langle v_{x}^{2}\right\rangle+\left\langle v_{y}^{2}\right\rangle+\left\langle v_{z}^{2}\right\rangle=3 v_{0}^{2}=3 k_{\mathrm{B}} T / M$ is the average centre of mass square velocity, and $M$ is the mass of a water molecule.

Experiments and MD results show that the translational harmonic motion of a water molecule in the cage gives rise to two peaks in $Z_{T}(\omega)$ at about 10 and $30 \mathrm{meV}$, respectively [42]. Thus, the following Gaussian functional form is used to represent approximately the translational part of the density of states:

$$
Z_{T}(\omega)=(1-C) \frac{\omega^{2}}{\omega_{1}^{2} \sqrt{2 \pi \omega_{1}^{2}}} \exp \left[-\frac{\omega^{2}}{2 \omega_{1}^{2}}\right]+C \frac{\omega^{2}}{\omega_{2}^{2} \sqrt{2 \pi \omega_{2}^{2}}} \exp \left[-\frac{\omega^{2}}{2 \omega_{2}^{2}}\right] .
$$

Moreover, the fit of MD results using equation (7) gives $C=0.44, \omega_{1}=10.8 \mathrm{THz}$, and $\omega_{2}=42.0 \mathrm{THz}$.

Using equations (5)-(7), we finally get an explicit expression for $F_{T}^{s}(Q, t)$ :

$$
F_{T}^{s}(Q, t)=\exp \left\{-Q^{2} v_{0}^{2}\left[\frac{1-C}{\omega_{1}^{2}}\left(1-\mathrm{e}^{-\omega_{1}^{2} t^{2} / 2}\right)+\frac{C}{\omega_{2}^{2}}\left(1-\mathrm{e}^{-\omega_{2}^{2} t^{2} / 2}\right)\right]\right\} .
$$

Equation (8) is the short-time behaviour of the translational ISF. It starts from unity at $t=0$ and decays rapidly to a flat plateau determined by an incoherent Debye-Waller factor $A(Q)$, given by

$$
A(Q)=\exp \left\{-Q^{2} v_{0}^{2}\left[\frac{1-C}{\omega_{1}^{2}}+\frac{C}{\omega_{2}^{2}}\right]\right\}=\exp \left[-Q^{2} a^{2} / 3\right]
$$




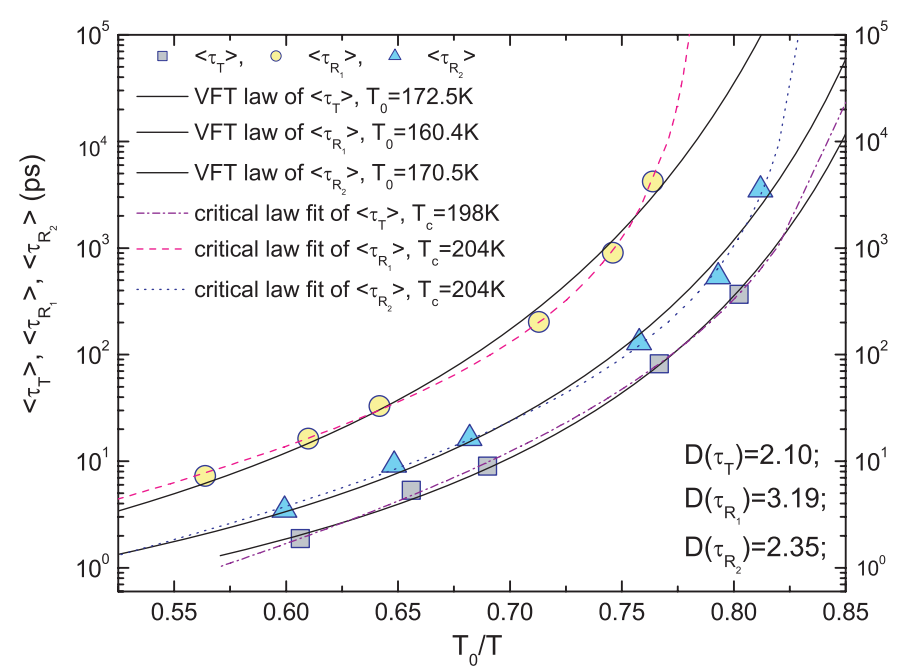

Figure 4. Temperature dependence of the average translational relaxation time, $\left\langle\tau_{T}\right\rangle$, and the average first- and second-order rotational correlation times, $\left\langle\tau_{R_{1}}\right\rangle$ and $\left\langle\tau_{R_{2}}\right\rangle$ respectively, as extracted from SPC/E bulk water MD data. It should be noted that, at the given temperature, $\left\langle\tau_{R_{1}}\right\rangle>\left\langle\tau_{R_{2}}\right\rangle>\left\langle\tau_{T}\right\rangle$ generally. The fitting curves, solid lines and dotted lines, show that for $T$ far above $T_{0}$, both the VFT law and critical law agree well with simulation data. However, as one approaches $T_{0}$, their difference begins to become apparent.

where $a$ is the root mean square vibrational amplitude of the water molecules in the cage, in which the particle is constrained during its short-time movements. According to MD simulations, $a \approx 0.5 \AA$ is fairly temperature independent [12].

On the other hand, the cage relaxation at long times can be described by the standard $\alpha$ relaxation model, according to the mode-coupling theory (MCT), with a stretched exponential having a structural relaxation time $\tau_{T}$ and a stretch exponent $\beta$. Therefore, the translational ISF, valid for the entire time range, can be written as a product of the short-time part and a long-time part:

$$
F_{T}(Q, t)=F_{T}^{s}(Q, t) \exp \left[-\left(t / \tau_{T}\right)^{\beta}\right] .
$$

The fit of the MD generated $F_{T}(Q, t)$ using equation (10) shows that $\tau_{T}$ is $Q$-dependent, obeying the power law

$$
\tau_{T}=\tau_{0}(a Q)^{-\gamma},
$$

where $\gamma$ is $\leqslant 2$, with a slight dependency on $Q$, and $\beta<1$ is slightly $Q$-dependent as well. In the $Q \rightarrow 0$ limit, one should approach the diffusion limit, where $\gamma \rightarrow 2$ and $\beta \rightarrow 1$. Thus the translational ISF can be written as $F_{T}(Q, t)=\exp \left[-D Q^{2} t\right], D$ being the self-diffusion coefficient. In QENS experiments, this low- $Q$ limit is not usually reached, and both $\beta$ and $\gamma$ can be considered $Q$-independent in the limited $Q$-range of $0-1[38,39]$.

Figure 4 shows that the average translational relaxation time from MD simulation with the SPC/E model, $\left\langle\tau_{T}\right\rangle=\left(\tau_{0} / \beta\right) \Gamma(1 / \beta)$, obeys both the VFT law and the critical law. It is well known that the diffusion coefficient of bulk water follows a critical behaviour [4]. In our case, at low temperature, we cannot extract the diffusion coefficient from the average translational relaxation time, $\left\langle\tau_{T}\right\rangle$, because it is not $Q^{2}$-dependent. The obtained data follow the critical law, which is in accordance with the reported temperature dependence of the self-diffusion coefficient for SPC/E water [13]: $D \sim(T / 198.7-1)^{2.73}$. Both $D$ and $\left\langle\tau_{T}\right\rangle$ follow a critical law in temperature, with similar $T_{\mathrm{c}}$. Since the SPC/E water temperature of maximum density 
is $T=250 \mathrm{~K}$, there is a $27 \mathrm{~K}$ offset between the real and computer temperature. Therefore, the critical temperature obtained by MD simulations in this case corresponds to $T \approx 225 \mathrm{~K}$ in real water. Meanwhile, the VFT law shows a good fit to the MD generated $\left\langle\tau_{T}\right\rangle$ as well. The VFT law is a way to fit relaxation times close to the glass transition point. In the VFT law, equation (1), $T_{0}$ is an ideal glass transition temperature. It is shown in figure 4 that $T_{0}=172.5 \mathrm{~K}$, corresponding to $200 \mathrm{~K}$ in bulk water.

Similarly derived $\left\langle\tau_{R_{1}}\right\rangle$ and $\left\langle\tau_{R_{2}}\right\rangle$, which are the first-order and second-order rotational correlation functions, are also shown in figure 4 with the critical law fit and VFT law fit. Since our QENS data analysis only takes into account the translational ISF, we do not show how to theoretically calculate the rotational correlation functions. However, details can be found in [40].

Combining equations (3), (8), and (10), we can calculate the theoretical value of $S_{\mathrm{H}}(Q, \omega)$ and compare it directly with its experimental value.

\section{Data analysis}

When we use the RCM to analyse spectra from QENS experiments, we usually observe an additional presence of a pronounced elastic component superimposed on the top of quasielastic broadening [40]. The presence of this elastic component is clearly detectable at most temperatures and $Q \mathrm{~s}$, even with the broader resolution of the DCS.

Defining the magnitude of the wavevector transfer, $Q$, corresponding to the scattering angle $\theta$ at the elastic channel as $Q_{0}=[4 \pi \sin (\theta / 2)] / \lambda$, we can analyse the experimental data according to the following model:

$$
S(Q, \omega)=p R\left(Q_{0}, \omega\right)+(1-p) F T\left\{F_{\mathrm{H}}(Q, t) R\left(Q_{0}, t\right)\right\}
$$

where $p$ is the fraction of the elastic component, $F_{\mathrm{H}}(Q, t) \approx F_{T}(Q, t)$ is the ISF of hydrogen atoms which defines the quasielastic scattering, and $R\left(Q_{0}, t\right)$ is the Fourier transform of the experimental resolution function, $R\left(Q_{0}, \omega\right)$.

Taking the weight of the elastic component as a fitting parameter, we obtain the temperature-dependent values for $p$. However, in this way we were trying to evaluate contributions from: (i) the hydrogen atoms of surface silanol groups; (ii) the water molecules interacting strongly with surface silanol groups; (iii) and some of the very slowly moving bulk water molecules near the surface.

(i) The elastic contribution is mainly due to the scattering of hydrogen atoms in surface silanol ( $\mathrm{Si}-\mathrm{O}-\mathrm{H})$ groups [17]. The dangling $\mathrm{O}$ and $\mathrm{H}$ atoms of water molecules are connected to the surface $\mathrm{Si}$ atom to form a silanol group. In a silanol group, the $\mathrm{H}$ atom is not fixed, but is constrained to move in a circle on the surface to keep the $\mathrm{O}-\mathrm{H}$ bond length and the $\mathrm{Si}-\mathrm{O}-\mathrm{H}$ angle constant.

(ii) It is known that for the hydroxylated silica surface, surface solvent hydrogen bonding is stronger than interactions in the bulk solvent, with the nearest solvent layer interacting specifically with up to three surface hydroxyl groups.

(iii) As the temperature decreases, the dynamics of water slows down markedly, and as a result, it is more difficult to resolve the quasielastic component even by the highest resolution QENS spectrometer. The bulk of the fluctuations are so slow that they could be seen as immobile by the QENS spectrometers and contributing to the elastic component at very low temperatures. So that the elastic contribution increases as the temperature decreases.

For temperatures lower than $270 \mathrm{~K}$, the fraction of the elastic component seems to increase, whereas for $T \geqslant 270 \mathrm{~K}$ a plateau is reached. The fast increase on lowering the temperature is obviously connected to the slowing down of water dynamics. In the meantime, the plateau 

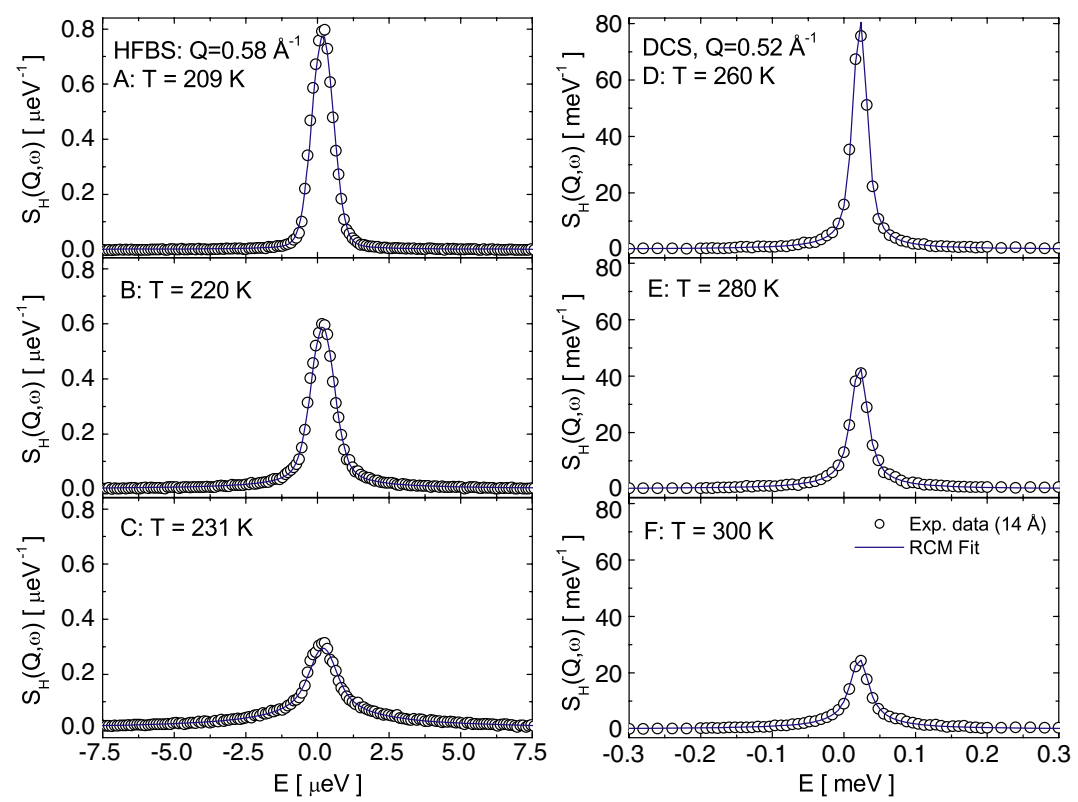

Figure 5. These typical QENS spectra of a hydrated MCM-41-S-14 sample show that the RCM analysis agrees well with experimental data. The left-hand panels show the spectra taken from the HFBS with a resolution of $0.8 \mu \mathrm{eV}$. The right-hand panels are the data taken from the DCS with a resolution of $20 \mu \mathrm{eV}$. The solid circles are the experimental data; the continuous lines, the RCM fit.

value is then the scattering due to the hydrogen atoms of surface silanol groups, without the contribution of bulk water and the water molecules interacting strongly with surface silanol groups. The surface silanol groups are so well organized and strong that they may be seen as temperature independent. For this reason, to obtain consistent results and only take into account the contribution from the surface silanol groups as the elastic component, we have fixed $p$ to its plateau value at high temperatures [40], listed in table 1, when analysing the data from different spectrometers.

The quasielastic broadening has been analysed according to the RCM as described in the previous section. $F_{\mathrm{H}}(Q, t)$ is described in terms of seven parameters (from equations (3), (8), (10), (11), and (12)): $C, \omega_{1}, \omega_{2}, \tau_{0}, \gamma, \beta$, and $p$. Three of them are related to the short-time dynamics, namely $C, \omega_{1}$, and $\omega_{2}$. The short-time dynamics is not strongly temperature dependent, according to MD simulation results. On the other hand, the quasielastic broadening is mostly determined by the long-time dynamics. Therefore, the values of $C, \omega_{1}$, and $\omega_{2}$ were fixed according to the MD simulation results [40], which were tested by the experimental results from INS with a satisfactory agreement $[10,11]$.

The remaining four parameters, namely $p, \tau_{0}, \gamma$, and $\beta$, could then be determined from the analysis of a group of low- $Q$ QENS spectra. We report the results of our analysis with the values of $p$ fixed to their plateau values.

We show in figure 5, as an example, two sets (temperature series) of QENS areanormalized spectra taken at the HFBS and DCS spectrometers. As can be seen, RCM analysis agrees well with experimental spectra in all investigated cases. It is remarkable that using four parameters we were able to reproduce the data from nine (DCS), and seven (HFBS) constant angle spectra. On the other hand, it is clearly shown in the figure that the quasielastic broadening is strongly temperature dependent. The width of the peak is 


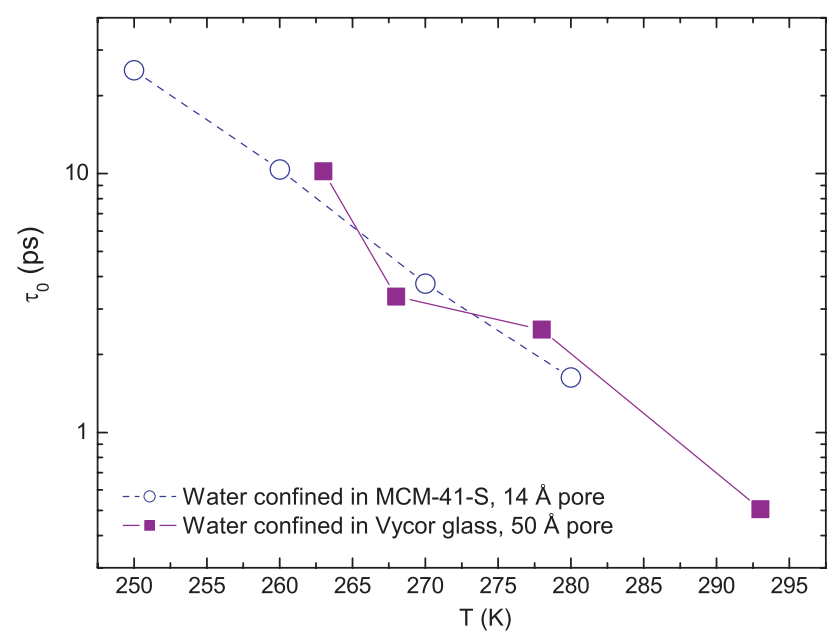

Figure 6. Comparison of the $Q$-independent translational relaxation time parameter $\tau_{0}$ (see equation (11)) for water confined in MCM-41-S-14 and water confined in Vycor glass with $50 \AA$ pore size, which is closer to bulk water. The latter is taken by using equation (11) to fit the data in [18]. It is seen that the two sets of data agree with each other within the experimental error in the region of temperatures where they overlap. The only difference in the two cases is the limit to which water can be supercooled without freezing.

progressively sharpened as the temperature is lowered. Thus for $T \leqslant 240 \mathrm{~K}$, the $0.8 \mu \mathrm{eV}$ resolution of the HFBS is necessary to obtain useful data.

It is likely that the water confined in a capillary, only a few molecular diameters wide, has physical properties different from those of bulk water. In particular, its structure is significantly affected by the shell formation and the hydrogen bonding in the outer layer (the outer layer means the layer approximately $5 \AA$ close to the surface). However, the main features of the $\mathrm{RCM}$ are the separation of the short-time and long-time dynamics and the generalization to a stretched exponential relaxation behaviour at long times, represented by the $\alpha$-relaxation. The short-time dynamics is calculated according to the harmonic approximation, taking into account the experimentally observed structure in the density of states of hydrogen atoms both in bulk water and in confined water in Vycor glass [11, 12,15]. The use of a stretched exponential relaxation function at long times accounts for the experimentally observed $\alpha$-relaxation in supercooled bulk water and water in Vycor glass [42]. In fact, MD simulation of water confined in $40 \AA$ radius Vycor pores clearly shows the existence of a two-step relaxation, namely a shorttime $\beta$-relaxation followed by a long-time $\alpha$-relaxation $[15,16]$. Therefore, the main features of the RCM model should be applicable to water confined in nanoporous matrices as well as in bulk supercooled water.

Experimentally, we show in figure 6 a comparison of water confined in two systems. One is MCM-41-S-14 with $14 \AA$ pore diameter, and the other one is a Vycor glass with $50 \AA$ pore diameter, closer to bulk water. The latter is taken by using equations (10) and (11) to fit the data in [18]. We can only compare $\tau_{0}$, instead of $\left\langle\tau_{T}\right\rangle$, because the $Q$-ranges covered in the two experiments are different. It is seen that the two sets of data agree with each other well within the experimental error in the overlapping region of the temperatures. The only difference between the two cases is the limit to which water can be supercooled without freezing. The conclusion here is that as far as the relaxation times in the picosecond and nanosecond range are concerned, the difference between the confined water in nanopores and bulk-like water in Vycor is not detectable. 

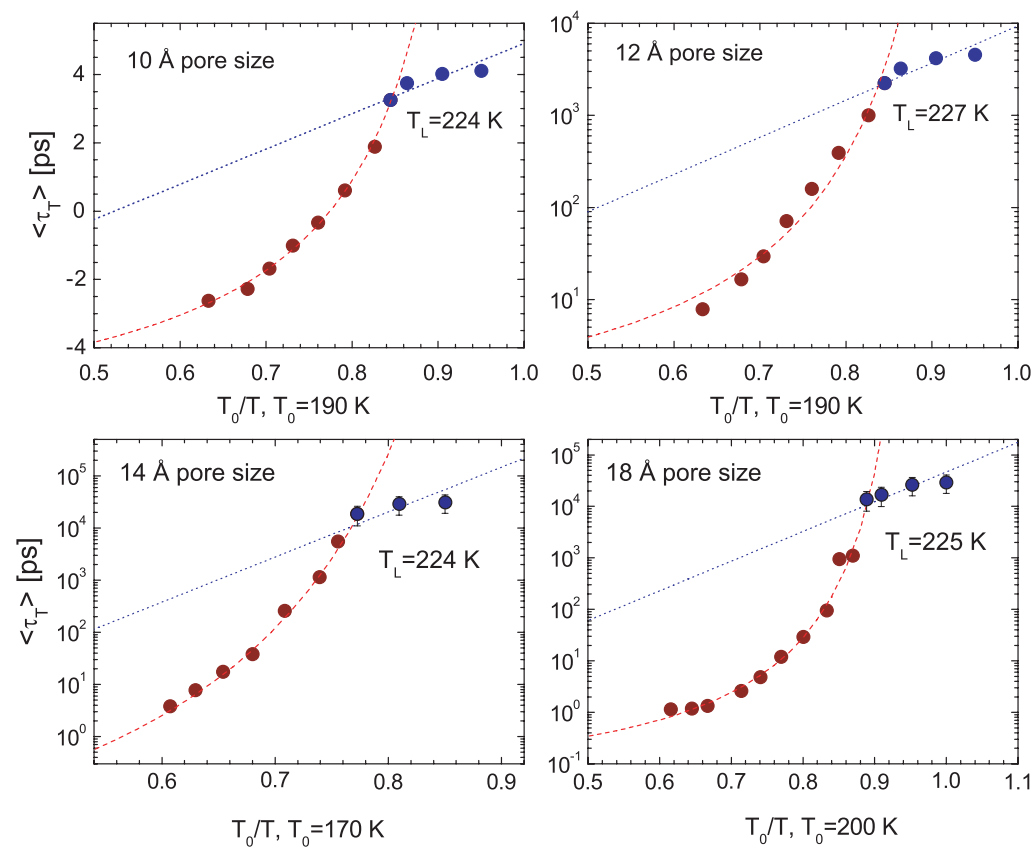

Figure 7. Temperature dependence of $\left\langle\tau_{T}\right\rangle$ plotted in $\log \left(\left\langle\tau_{T}\right\rangle\right)$ versus $T_{0} / T$ scale. Data at ambient pressure from $\mathrm{H}_{2} \mathrm{O}$ confined in MCM-41-S with different pore sizes are shown in different panels. Solid circles are the experimental data; the dashed lines are the VFT law fit of the experimental data; and the dotted lines are the Arrhenius law fit of the experimental data.

\section{Results}

The fitting of the data allowed us to extract parameters describing the translational dynamics of water, and calculate the average translational relaxation time, $\left\langle\tau_{T}\right\rangle$, which will show the FS crossover phenomenon, and that is the central result of this paper.

Figure 7 shows the temperature dependence of the average translational relaxation times, $\left\langle\tau_{T}\right\rangle=\frac{\tau_{0}}{\beta} \Gamma(1 / \beta)$, where $\Gamma$ is the gamma function, and $\tau_{0}$ and $\beta$ are the characteristic relaxation time and the stretch exponent of the translational dynamics, respectively. The resultant $\left\langle\tau_{T}\right\rangle$ has been fitted to a VFT law, for $T \geqslant 230 \mathrm{~K}$, obtaining parameters, $D, T_{0}$, and $\tau_{1}$, for different pore sizes at ambient pressure (listed in table 2). A critical law, which is usually used for bulk supercooled water, would fit the data above $240 \mathrm{~K}$, but deviates from the data below $240 \mathrm{~K}$ [43]. At $T \leqslant 225 \mathrm{~K}$, there is a sudden change in the slope of the $1 / T$-dependence of $\log \left(\left\langle\tau_{T}\right\rangle\right)$, which can be fitted with an Arrhenius law, with activation energies $E_{\mathrm{A}}$ reported in table 2. Our best fit to $\left\langle\tau_{T}\right\rangle$ gives us $T_{0}$ values in the vicinity of $200 \mathrm{~K}$, and $D$ values around 2 , indicating a very fragile liquid. Our finding is in agreement with the known properties of water below the melting point and in the supercooled region. In fact, a fragile-to-strong (FS) crossover in water was proposed [2] on the basis of the determination of the fragility of water near the melting and glass transition temperatures: near $T_{\mathrm{g}}$ water is a very strong liquid, whereas in the supercooled region it is the most fragile one. A strong experimental support for this hypothesis has already been given by a dielectric relaxation investigation of water confined in vermiculite clay [3], although the actual transition was not observed. It is to be noted that the crossover temperature $T_{\mathrm{L}}$ for different pore size samples, reported in figure 7 and table 2, does not change within error bars. Thus we propose that when the pore size of confinement is sufficiently small, $\leqslant 18 \AA$, 


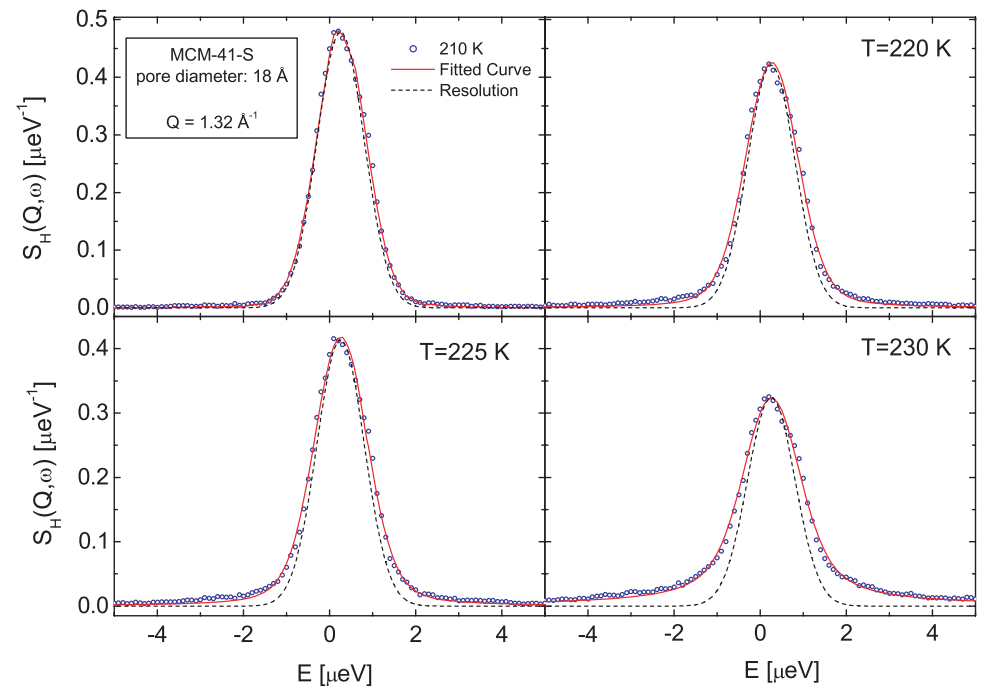

Figure 8. A temperature series of QENS spectra taken from the HFBS relative to their resolution functions are shown in this figure. The four temperatures are 210,220, 225, and $230 \mathrm{~K}$, which are close to the proposed fragile-to-strong transition temperature. The squares represent the real data taken from experiments; the solid lines represent the result of the fit; and the dashed lines represent the Gaussian resolution function.

Table 2. Fitted parameters of experimental data taken at ambient pressure.

\begin{tabular}{llllll}
\hline Samples & $T_{0}(\mathrm{~K})$ & $\mathrm{D}$ & $\tau_{1}(\mathrm{ps})$ & $E_{\mathrm{A}}\left(\mathrm{kcal} \mathrm{mol}^{-1}\right)$ & $T_{\mathrm{L}}(\mathrm{K})$ \\
\hline Mac-41-S-10 & 190 & 1.58 & 0.0045 & 3.91 & 224 \\
Mac-41-S-12 & 190 & 1.51 & 0.0086 & 3.51 & 227 \\
Mac-41-S-14 & 200 & 1.31 & 0.2036 & 4.81 & 224 \\
Mac-41-S-18 & 200 & 1.47 & 0.079 & 5.28 & 225 \\
\hline
\end{tabular}

the FS crossover temperature is size independent, which means that FS crossover is a universal property of supercooled water when the length scale of confinement is of order of nanometre size.

On the other hand, we report in figure 8 the spectra collected in the region of the FS crossover for fully hydrated MCM-41-S-18. The sharpening of the peak from $T=230$ to $225 \mathrm{~K}$, being more noticeable than the one from 220 to $210 \mathrm{~K}$, already suggests the existence of a crossover at $225 \mathrm{~K}$. The normalized data were analysed using equation (12). Also to be noted is that the broadening of the experimental data over the resolution function, shown in all four panels of figure 8, leaves enough dynamic information to be extracted by the RCM.

Before discussing the results from QENS experiments under pressure, figure 9 draws, as an example, two complete sets (temperature series) of QENS area-normalized spectra. The broadening of the quasi-elastic peaks becomes more and more noticeable as temperature increases. In figure 9(A), we may notice, from the shoulders of these spectral lines, that two groups of curves, 231-251 K and 199-209 K, are separated by the curve at a temperature of $220 \mathrm{~K}$. This visual information reinforces the result of the detailed analysis shown in figures 10 and 11 , that there is an abrupt dynamical crossover at $T_{\mathrm{L}}=219 \mathrm{~K}$, at 400 bar pressure. Figure $9(\mathrm{C})$ shows the RCM analysis of the spectrum taken at $T=220 \mathrm{~K}$, close to the crossover temperature. On the other hand, in figure 9(B), the spectra at pressure 2000 bar show a rather 

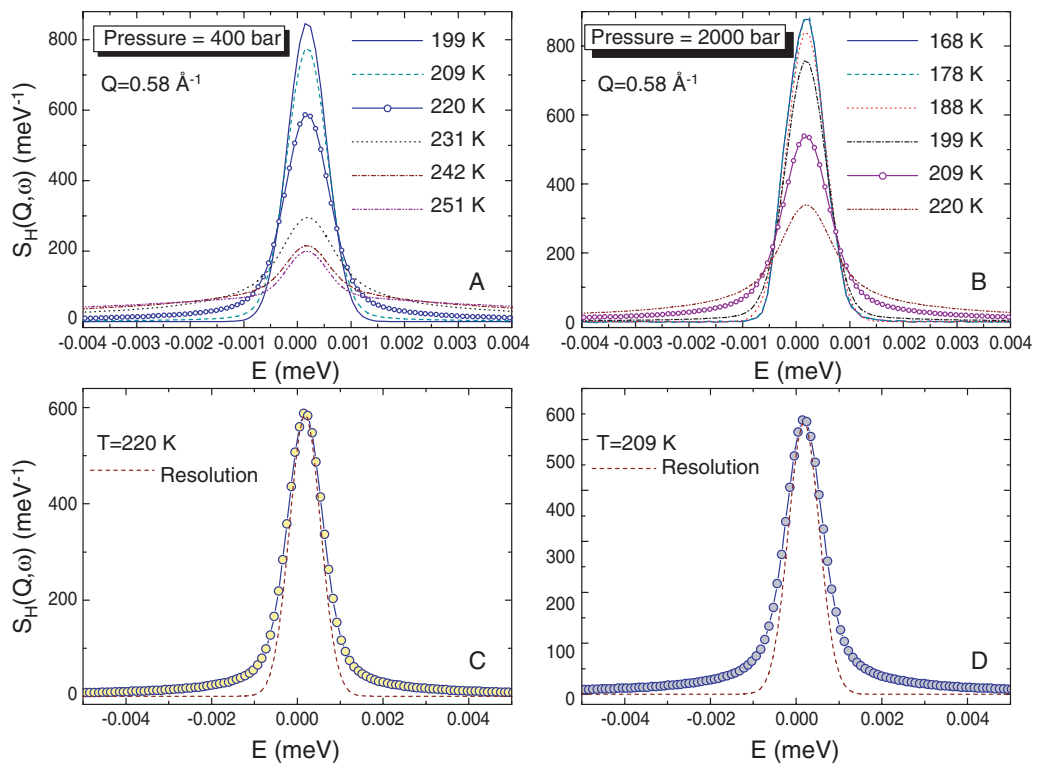

Figure 9. Spectra illustrating the difference between the presence (left panels) and the absence (right panels) of well-defined dynamic crossover as a function of temperature. The top panels A and B show QENS spectra measured at $Q=0.58 \AA^{-1}$, at two pressures, 400 bar and 2000 bar, and at a series of temperatures. The bottom panels $\mathrm{C}$ and D show the RCM analysis of one of the spectra from each pressure. The resolution function in each case is shown by a dashed line.

smooth variation with temperature, indicating that there is no sharp transition. Figure 9(D) is, again, an RCM analysis of the spectrum taken at $T=209 \mathrm{~K}$ of this pressure. The RCM, as one can see, reproduces well the experimental spectral line shapes of confined water. The broadening of the experimental data over the resolution function, shown in figures 9 (C) and (D), leaves enough dynamic information to be extracted by RCM.

We have already shown that at ambient pressure, for fully hydrated MCM-41-S of pore size $\leqslant 18 \AA,\left\langle\tau_{T}\right\rangle$ exhibits an FS crossover with the same crossover temperature $T_{\mathrm{L}}=225 \mathrm{~K}$ within error bars. In figures 10 and 11 , we exhibit the temperature variation of $\left\langle\tau_{T}\right\rangle$ for water molecules as a function of pressure. It is seen that the panels in figure 10 all clearly show an FS crossover from a VFT law to an Arrhenius law. This crossover is the signature of an FS dynamic transition predicted by Ito et al [2], and now extends into finite pressures. The transition temperature, $T_{\mathrm{L}}$, as the crossing point of the VFT law and Arrhenius law, is calculated by $1 / T_{\mathrm{L}}=1 / T_{0}-\left(D k_{\mathrm{B}}\right) / E_{\mathrm{A}}$. All the parameters for the VFT and Arrhenius law fitting are reported in table 3. However, in figure 11, the cusp-like transition becomes rounded off and there is no clear-cut way of defining the FS crossover temperature. Note that while we have done more measurements at high temperatures at 2000 bar pressure, shown in figure 11(B), there is still a hint of fragile behaviour at high enough temperature.

As far as the parameters obtained from the fit are concerned, figure 12 reports the fitted plateau value of $p$ for different pressures. Figure 13 reports the temperature dependence of the product $\beta \gamma$ for a pressure of 800 bar. In the measured temperature range and similar to the one taken at ambient pressure [43], $\beta \gamma$, the actual exponent of the $Q$-dependence of the ISF, does not reach 2 , as in the free diffusion case, even at room temperature. The value of $\beta \gamma$ in figure 13 starts from a plateau value 1.3 at $320 \mathrm{~K}$, close to the value for ambient pressure. Then it decreases gradually until $T \approx 210 \mathrm{~K}$ and it reaches another plateau at about 0.7 . This 

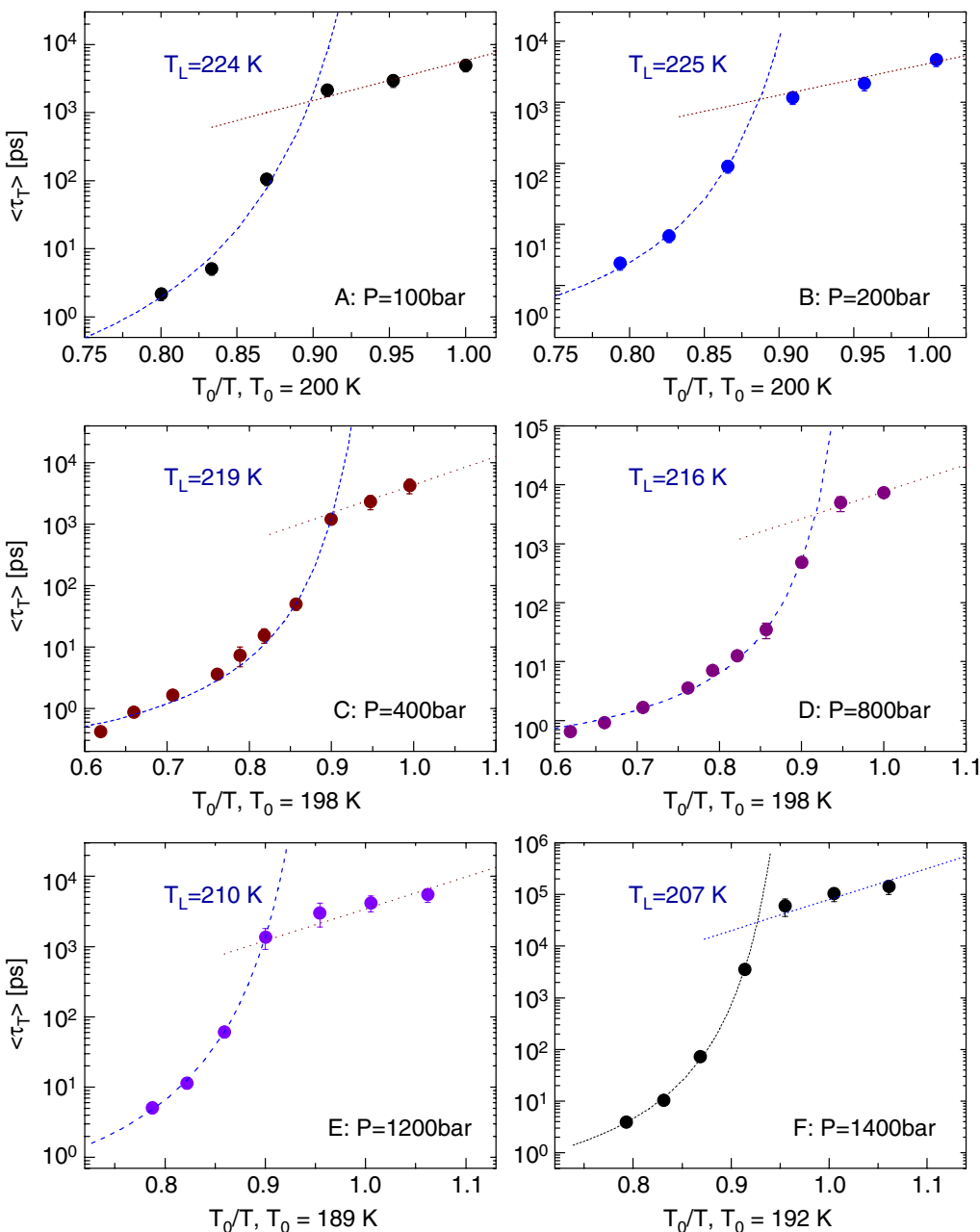

Figure 10. Summary of the data from the pressure range where a well-defined fragile-to-strong crossover is observed. Temperature dependence of $\left\langle\tau_{T}\right\rangle$ plotted in $\log \left(\left\langle\tau_{T}\right\rangle\right)$ versus $T_{0} / T$ scale. Data at 100, 200, 400, 800, 1200, 1400 bar are shown in panels A, B, C, D, E, and F, respectively.

Table 3. Fitted parameters of QENS experiments taken at applied pressures; sample: fully hydrated MCM-41-S-14.

\begin{tabular}{llllll}
\hline Pressures (bar) & $T_{0}(\mathrm{~K})$ & $D$ & $\tau_{1}(\mathrm{ps})$ & $E_{\mathrm{A}}\left(\mathrm{kcal} \mathrm{mol}^{-1}\right)$ & $T_{\mathrm{L}}(\mathrm{K})$ \\
\hline 100 & 200 & 1.38 & 0.008 & 5.56 & 224 \\
200 & 207 & 0.929 & 0.026 & 4.78 & 225 \\
400 & 198 & 1.004 & 0.111 & 4.16 & 219 \\
800 & 198 & 0.859 & 0.202 & 4.15 & 216 \\
1200 & 189 & 1.05 & 0.097 & 3.94 & 210 \\
1400 & 192 & 1.00 & 0.082 & 5.26 & 207 \\
\hline
\end{tabular}

drop, not as precipitous as that at ambient pressure [40], also signals a change of the dynamical behaviour of water at $210-220 \mathrm{~K}$, signifying that water is structurally arrested. 


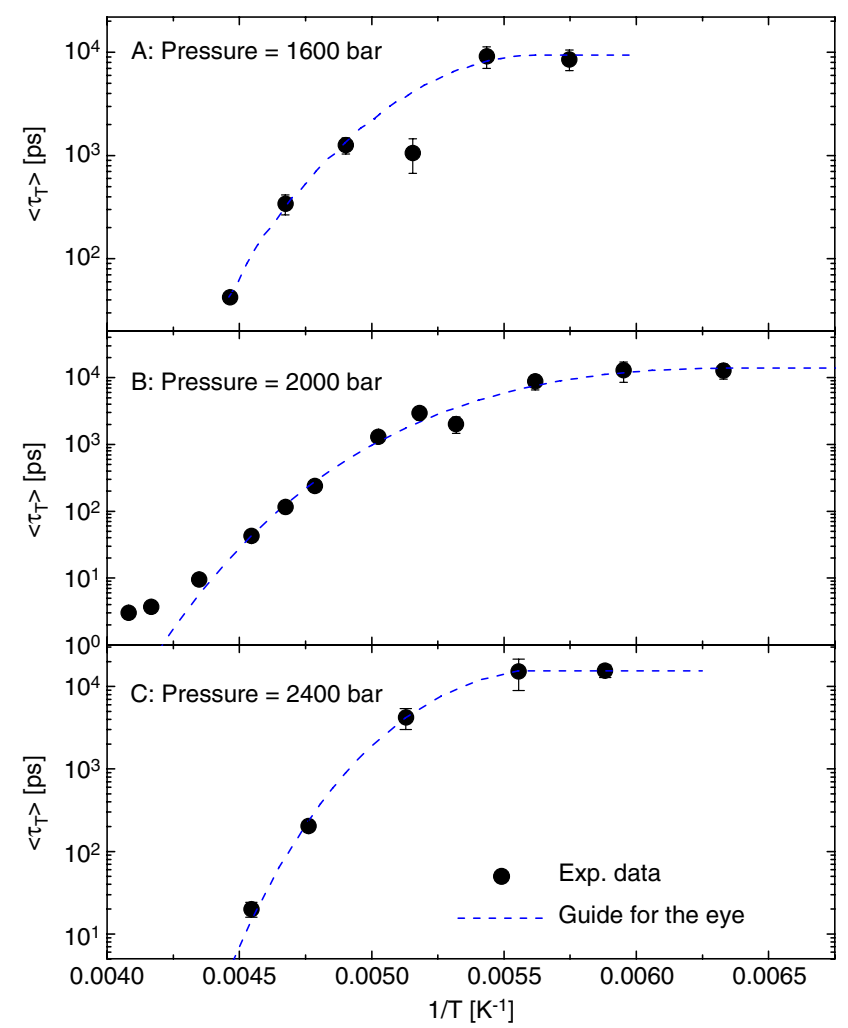

Figure 11. Summary of the data from the pressure range where no well-defined fragile-to-strong crossover is observed. Temperature dependence of $\left\langle\tau_{T}\right\rangle$ plotted in $\log \left(\left\langle\tau_{T}\right\rangle\right)$ versus $1 / T$ scale. Data at 1600, 2000, and 2400 bar are shown in panels A, B, and C, respectively.

To determine the state of water before and after FS crossover, HRMECS measurements of INS were performed on $55 \mathrm{wt} \%$ water confined in MCM-41-S with $18 \AA$ pore size. Figure 14 shows the observed vibrational density of states (DOS) of confined water at different temperatures as compared with those of ice Ih. It should be noted that the $G(E)$ of ice (solid line) is characterized by a much steeper leaning edge compared to those of supercooled water in the temperature range from 210 to $240 \mathrm{~K}$. The broader $G(E)$ of supercooled water confined in MCM-41-S with pore diameter of $18 \AA$ is seen to be more characteristic of a liquid state. From the behaviour of the low-energy cut-off of the librational band around $50 \mathrm{meV}$, it is obvious that the state of confined water (its hydrogen bond network) is different at temperatures above and below $225 \mathrm{~K}$. The difference between the average spectra above and below $225 \mathrm{~K}$ is shown to have a clear peak at around $50 \mathrm{meV}$ (dash-dot-dot line). It is obvious from the figure that the confined water does not transform into ice at all these temperatures.

In addition, more results from INS measurements using the HRMECS taken at pressure 800 bar and 1600 bar are reported in figure 15. From the behaviour of the leaning edge of the librational band near $50 \mathrm{meV}$ energy transfer at 800 bar pressure, it is obvious that the state of confined water is different at temperatures above and below $220 \mathrm{~K}$, and forms two groups: 200-210 K and 230-240 K. This separation of leaning edge into two groups signals a nonuniform dynamical transition of water at around $220 \mathrm{~K}$, near $T_{\mathrm{L}}$ of this pressure. However, at a pressure above $p_{\mathrm{C}}$ at 1600 bar, the dynamical transition is smooth (shown by figure 11), so there are no detectable groups formed in the experimental temperature range. 


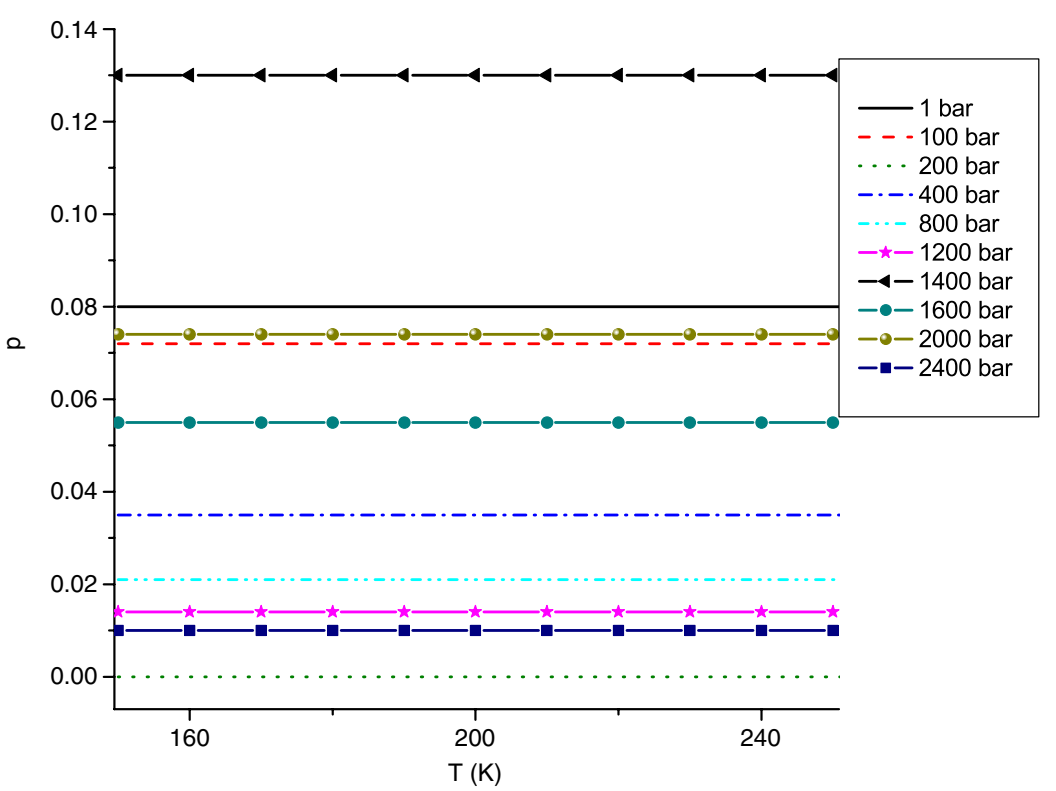

Figure 12. Fitted parameter of the fraction of the elastic component $p$ value at all pressures.

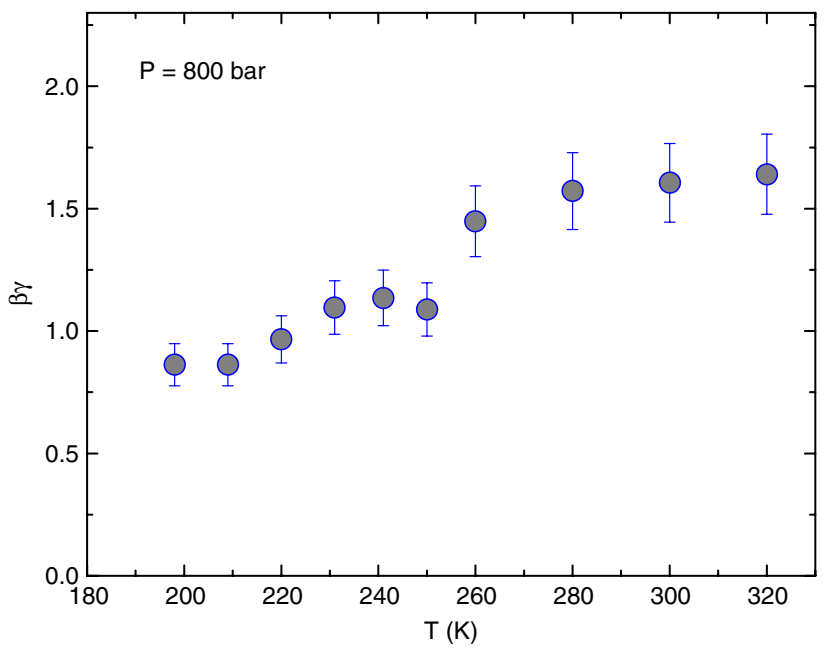

Figure 13. Temperature dependence of the product $\beta \gamma$ for a pressure of 800 bar, where $\beta$ is the stretch exponent, and $\gamma$ is the power law exponent controlling the $Q$-dependence of the translational relaxation time.

\section{Discussion: the liquid-liquid coexistence line and the associated Widom line}

According to the liquid-liquid phase transition hypothesis [44], to explain the anomalies of thermodynamic and transport properties of supercooled water, one postulates the existence of a first-order phase transition line between two phases of liquid water: a low-density liquid (LDL) and a high-density liquid (HDL). This line is called the liquid-liquid (L-L) coexistence line and it terminates at an $\mathrm{L}-\mathrm{L}$ critical point. It is to be noted that if the state point is on the 


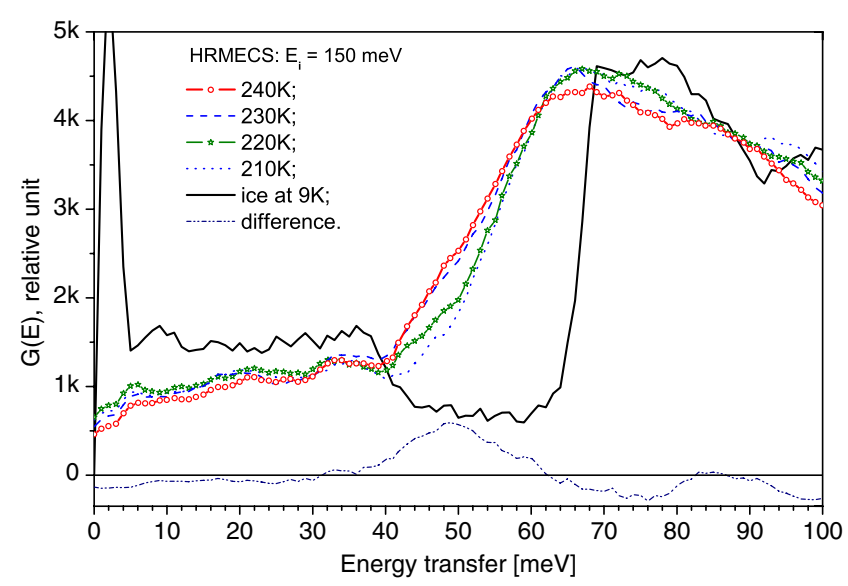

Figure 14. The generalized librational density of states $G(E)$ (taken with $Q<2 \AA^{-1}$ ) of ice and confined water (within the energy range from 40 to $120 \mathrm{meV}$ ) at different temperatures measured with the HRMECS using incident neutron energy of $150 \mathrm{meV}$. It should be noted that the $G(E)$ of ice (solid line) is characterized by a much steeper leaning edge compared to that of supercooled water in the temperature range from 210 to $240 \mathrm{~K}$. The broader $G(E)$ of supercooled water confined in MCM-41-S with pore diameter of $18 \AA$ is seen to be more characteristic of a liquid state. From the behaviour of the low-energy cut-off of the librational band around $50 \mathrm{meV}$, it is obvious that the state of confined water (its hydrogen bond network) is different at temperatures above and below $225 \mathrm{~K}$. The difference between the average spectra above and below $225 \mathrm{~K}$ is shown to have a clear peak at around $50 \mathrm{meV}$ (dash-dot-dot line).

L-L coexistence line, one has a two-phase liquid consisting of a mixture of HDL and LDL, just as the gas and liquid phases are coexisting on the liquid-gas coexistence line (refer to the $P-\rho$ diagram of superheated water, figure 2.2 of [45]). The L-L coexistence line extends into the one-phase region after terminating at the critical point [46]. This extended line, not being real, is the so-called Widom line or the critical isochore. The Widom line is defined as a straight line in the pressure-temperature $(P-T)$ plane, starting from the critical point $C^{*}\left(p_{C}, T_{C}\right)$ and extending into the one-phase region, with the same slope as that of the $\mathrm{L}-$ $\mathrm{L}$ coexistence line at $\left(p_{C}, T_{C}\right)$. Even though this line is an imaginary line, experiments on superheated water show that many thermodynamic quantities and transport coefficients, such as the isothermal compressibility, thermal-expansion coefficient, isobaric specific heat capacity, isochoric specific heat capacity, speed of sound, thermal conductivity, shear viscosity, and thermal diffusivity [45], show a peak when crossing the Widom line at a constant pressure.

Summarizing all the experimental results of fully hydrated MCM-41-S-14 under pressure, we show in a $P-T$ plane, in figure 16 [50], the observed pressure dependence of $T_{\mathrm{L}}$ and its estimated continuation, denoted by a dashed line, in the pressure region where no clear-cut FS crossover is observed. One should note that the $T_{\mathrm{L}}$ line has a negative slope, parallel to the TMD line, indicating a lower density liquid on the lower temperature side. This $T_{\mathrm{L}}$ line also approximately tracks the $T_{\mathrm{H}}$ line, and terminates in the upper end when intersecting the $T_{\mathrm{H}}$ line at $1600 \mathrm{bar}$ and $200 \mathrm{~K}$, at which point the character of the dynamic transition changes. We shall discuss the significance of this point later on. A special feature of the $T_{\mathrm{L}}$ line at the lower end should be noted as well. The line essentially becomes vertical after around 200 bar and the transition temperature approaches a constant value of $\sim 225 \mathrm{~K}$.

Since $T_{\mathrm{L}}$ determined experimentally is a dynamic crossover temperature, it is natural to question whether the system is in a liquid state on both sides of the $T_{\mathrm{L}}$, and if so, what the 


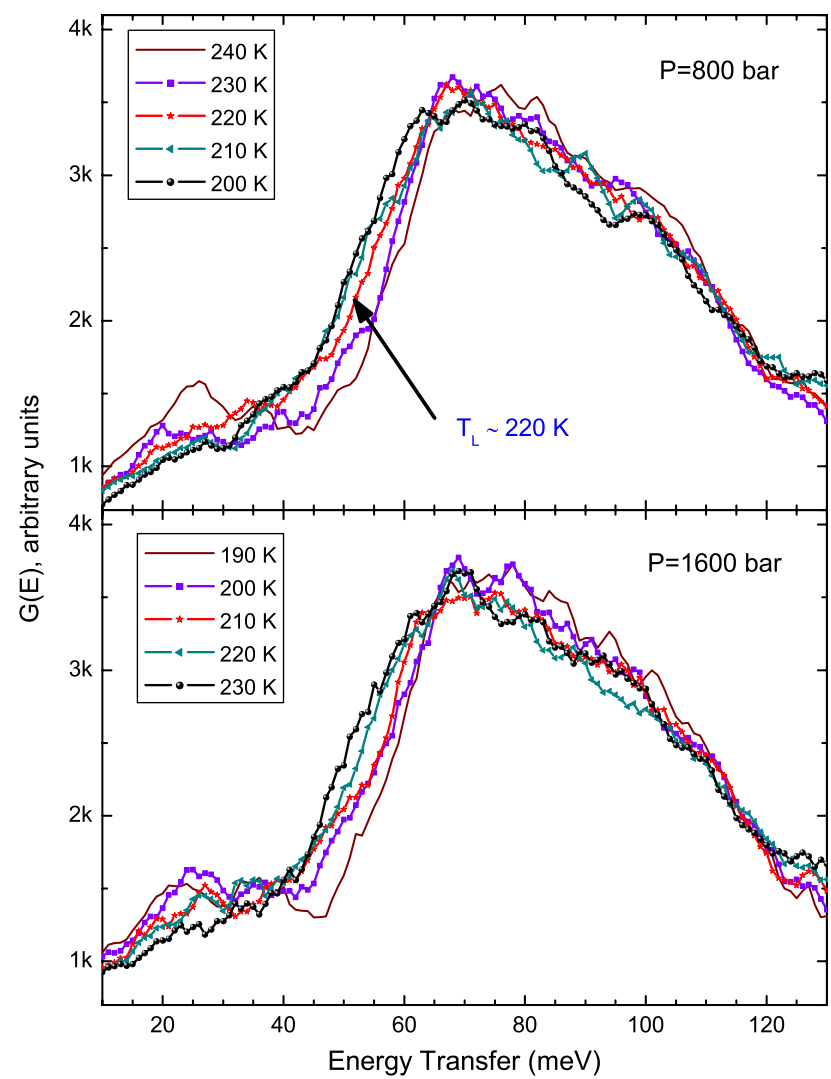

Figure 15. The comparison of two generalized librational density of states $G(E)$ (taken with $Q<2 \AA^{-1}$ ) of water confined in MCM-41-S-14 at a series of temperatures, taken at pressures below and above the critical pressure $p_{\mathrm{C}} \sim 1500$ bar (see figure 16).

nature of the high-temperature and low-temperature liquids would be. Sastry and Angell have recently shown by an MD simulation that at a temperature $T \approx 1060 \mathrm{~K}$ (at zero pressure), below the freezing point $1685 \mathrm{~K}$, the supercooled liquid silicon undergoes a first-order liquidliquid phase transition, from a fragile, dense liquid to a strong, low-density liquid with nearly tetrahedral local coordination [51]. Prompted by this finding, we would like to relate, in some way, our observed $T_{\mathrm{L}}$ line to the L-L transition line, predicted by MD simulations of water [52] and speculating on the possible location of the low-temperature critical point.

According to INS experiments, water remains in a disordered liquid state both above and below the FS crossover at ambient pressure (figure 14). Furthermore, our analysis of the FS crossover for the case of ambient pressure indicates that the activation energy barrier for initiating the local structural relaxation is $E_{\mathrm{A}}=4.89 \mathrm{kcal} \mathrm{mol}^{-1}$ for the low-temperature strong liquid. Yet, previous INS experiments of the stretch vibrational band of water [53] indicate that the effective activation energy of breaking a hydrogen bond at $258 \mathrm{~K}$ (high-temperature fragile liquid) is $3.2 \mathrm{kcal} \mathrm{mol}^{-1}$. Therefore, it is reasonable to conclude that the high-temperature liquid corresponds to the high-density liquid (HDL) where the locally tetrahedrally coordinated hydrogen bond network is not fully developed, while the low-temperature liquid corresponds to the low-density liquid (LDL) where the more open, locally ice-like hydrogen bond network is fully developed [54]. 


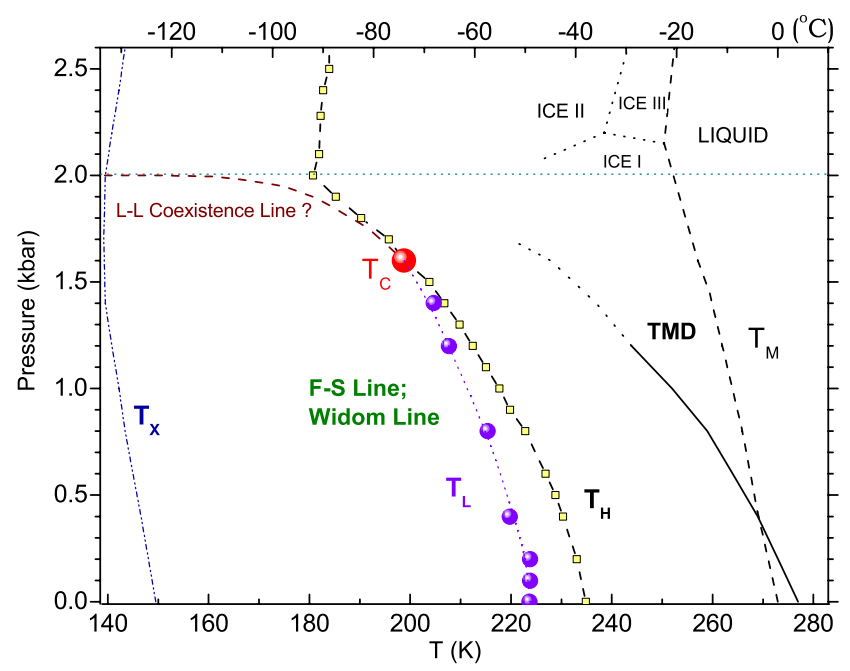

Figure 16. The pressure dependence of the measured FS dynamic crossover temperature, $T_{\mathrm{L}}$, plotted in the $P-T$ plane (solid circles). Also shown are the homogeneous nucleation temperature line, denoted as $T_{\mathrm{H}}$ [47], crystallization temperatures of amorphous solid water, denoted as $T_{\mathrm{X}}$ [48], and the temperature of maximum density line, denoted as TMD [49], taken from known phase diagram of bulk water.

It is appropriate now to address the possible location of the second critical point [52]. Above the critical temperature $T_{\mathrm{C}}$ and below the critical pressure $p_{\mathrm{C}}$, we expect to find a onephase liquid with a density $\rho$, which is constrained to satisfy an equation of state: $\rho=f(p, T)$. If an experiment is done by varying temperature $T$ at a constant pressure $p<p_{\mathrm{C}}, \rho$ will change from a high-density value (corresponding to the HDL) at sufficiently high temperature to a low-density value (corresponding to the LDL) at sufficiently low temperature. Since the fragile behaviour is associated with the HDL and the strong behaviour with the LDL, we should expect to see a clear FS crossover as we lower the temperature at this constant $p$. Therefore, the cusplike FS crossover we observed should then occur when we cross the so-called Widom line in the one-phase region [45]. On the other hand, if the experiment is performed in a pressure range $p>p_{\mathrm{C}}$, corresponding to the two-phase region and crossing the $\mathrm{L}-\mathrm{L}$ coexistence line, the system will be consisting of a mixture of different proportions of HDL and LDL as one varies $T$. In this latter case, the $\left\langle\tau_{T}\right\rangle$ versus $1 / T$ plot will not show a clear-cut FS crossover (the transition will be washed out) because the system is in a mixed state. The above picture would then explain the dynamical behaviour we showed in figures 10 and 11 . In figures 10 and 11, a clear FS crossover is observed up to 1400 bar, and beyond 1600 bar the crossover is rounded off. From this observation, the reasonable location of the $\mathrm{L}-\mathrm{L}$ critical point is estimated to be at $p_{\mathrm{C}}=1500 \pm 100$ bar and $T_{\mathrm{C}}=200 \pm 10 \mathrm{~K}$, shown by a big round point in figure 16 .

Additionally, in a recent MD simulation using TIP5P, ST2, and the Jagla Model Potential of $\mathrm{Xu}$ et al [55], a small peak was found in the specific heat $C_{\mathrm{P}}$ when crossing the Widom line at a constant $p$. Meanwhile, Maruyama et al conducted an experiment on the adiabatic calorimetry of water confined within nanopores of silica gel [56]. It was found that water within $30 \AA$ pores was well prevented from crystallization, and it also showed a small $C_{\mathrm{P}}$ peak at $227 \mathrm{~K}$ at ambient pressure. This experimental result further supports that the FS crossover we observed at $225 \mathrm{~K}$ at ambient pressure is caused by the crossing of the Widom line in the one-phase region above the critical point [55]. 


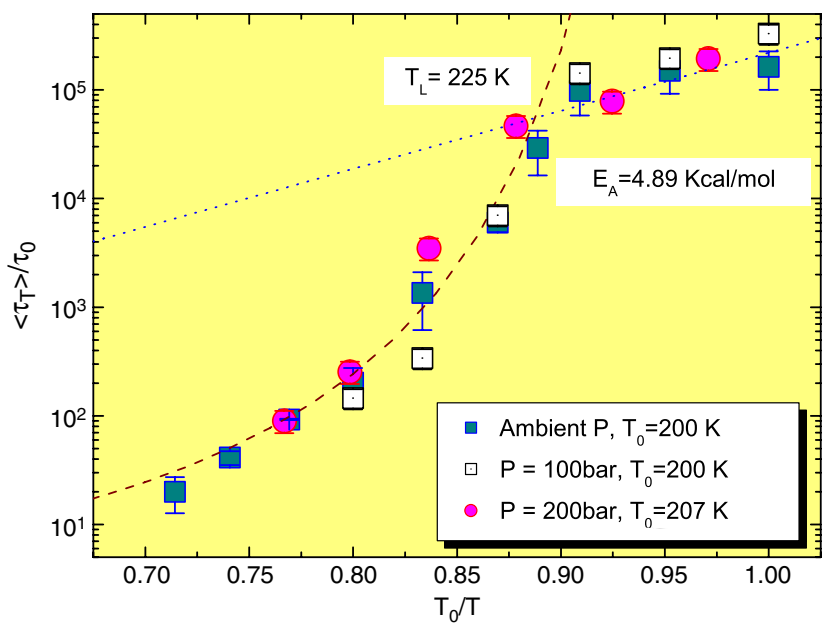

Figure 17. Scaling plot of $\log \left(\left\langle\tau_{T}\right\rangle / \tau_{0}\right)$ versus $T_{0} / T$ for data taken from 200, 100 and 1 bar. The fact that the three sets of data collapse into one master curve signifies that the slow dynamics becomes similar for pressures $\leqslant 200$ bar. The VFT and Arrhenius law fit of the master curve gives the transition temperature $T_{\mathrm{L}}=225 \mathrm{~K}$ and the activation energy $E_{\mathrm{A}}=4.89 \mathrm{kcal} \mathrm{mol}^{-1}$.

Moreover, we find that the long-time dynamics of water below 200 bar becomes similar, as noted in figure 17 . This special property may lead to another scenario, that there is one more critical point lying in the negative pressure range, as was proposed by Brovchenko et al (Brovchenko et al studied supercooled water using Monte Carlo simulation with different potential models: TIP4P, TIP5P, and SPC/E. All these models show two liquid-liquid phase transitions in the supercooled region. This result suggests the possibility of water having two low-temperature critical points, one at positive pressure and the other at negative pressure) [57]. Also, the critical point in the negative pressure region was found in the case of the MD simulated liquid silicon quoted above [51], and with symmetric position in contrast with the one we show in figure 16 referring to the ambient $(P, T)$ state point.

\section{Conclusion}

In conclusion, we have given a detailed account of our QENS studies of the dynamics of supercooled water confined in nanoporous silica materials, MCM-41-S, with different pore sizes and under pressure.

QENS data were analysed by using the RCM model previously developed by us. Four quantities were extracted from the analysis: they are $p, \tau_{0}, \beta$, and $\gamma$. In particular, we have discussed the temperature dependence of $\left\langle\tau_{T}\right\rangle=\frac{\tau_{0}}{\beta} \Gamma(1 / \beta)$ at ambient pressure and noted that the rate of dynamic slowing down changes abruptly at a temperature around $225 \mathrm{~K}$, signalling the onset of an avoided structural arrest transition of a high-density water into a low-density water. This FS dynamic crossover, then, has been determined for $\left\langle\tau_{T}\right\rangle$ at about $225 \mathrm{~K}$.

Moreover, we studied the slow dynamics of supercooled confined water under various applied pressures using HFBS and DCS at NIST NCNR. We observed clear evidence of a cusplike FS dynamic crossover at pressures lower than 1600 bar. We have shown, in this paper, that the crossover temperature decreases steadily with an increasing pressure, until it intersects the homogenous nucleation temperature line of bulk water at a pressure of 1600 bar. Above this pressure, it is no longer possible to discern the characteristic feature of the FS crossover. 
This paper has elaborated the detailed analysis of experimental data, and in that process, it has elucidated the new finding that the FS crossover is the result of crossing the Widom line at a constant pressure. We then estimated the location of the end point of the Widom line which should be the much anticipated second critical point of water. It is our future task to search for the locus of the $\mathrm{L}-\mathrm{L}$ coexistence line and establish experimentally the existence of the $\mathrm{L}-\mathrm{L}$ phase transition phenomena in water.

\section{Acknowledgments}

We are grateful for the technical support given to us by I Peral, J R D Copley and D A Neumann from NIST Center for Neutron Research. The work performed at ANL was supported by the US DOE-BES under contract No. W-31-109-ENG-38. Research at MIT is supported by DEFG02-90ER45429 and 2113-MIT-DOE-591. This work utilized facilities supported in part by the National Science Foundation under Agreement No. DMR-0454672. We benefitted from affiliation with EU supported Marie-Curie Research and Training Network on Arrested Matter.

\section{References}

[1] Angell C A 1991 J. Non-Cryst. Solids 131-133 13

[2] Ito K, Moynihan C T and Angell C A 1999 Nature 398492

[3] Bergman R and Swenson J 2000 Nature 403283

[4] Speedy R J and Angell C A 1976 J. Chem. Phys. 65851

[5] Chen S-H and Bellissent-Funel M-C 1994 Hydrogen Bond Networks (NATO Advances Study Institute, Series C: Mathematical and Physical Sciences vol 435) ed M-C Bellissent-Funel and J C Dore (Dordrecht: KluwerAcademic) p 289

[6] Zanotti J-M, Bellissent-Funel M-C and Chen S-H 2005 Eur. Phys. Lett. 7191

[7] Tarek M and Tobias D J 2002 Phys. Rev. Lett. 88138101

[8] Lynden-Bell R M and Rasaiah J C 1996 J. Chem. Phys. 1059266

[9] Settles M and Doster W 1996 Faraday Discuss. Chem. Soc. 103269

[10] Chen S-H, Liao C, Sciortino F, Gallo P and Tartaglia P 1999 Phys. Rev. E 596708

[11] Liu L, Faraone A and Chen S-H 2002 Phys. Rev. E 65041506

[12] Gallo P, Sciortino F, Tartaglia P and Chen S-H 1996 Phys. Rev. Lett. 762730

[13] Sciortino F, Gallo P, Tartaglia P and Chen S-H 1996 Phys. Rev. E 546331

[14] Chen S-H, Gallo P, Sciortino F and Tartaglia P 1997 Phys. Rev. E 564231

[15] Gallo P, Rovere M and Spohr E 2000 Phys. Rev. Lett. 854317

[16] Gallo P, Rovere M and Spohr E 2000 J. Chem. Phys. 11311324

[17] Lee S H and Rossky P J 1994 J. Chem. Phys. 1003334

[18] Zanotti J M, Bellissent-Funel M-C and Chen S-H 1999 Phys. Rev. E 593084

[19] Botti A, Bruni F, Isopo A, Ricci M A and Soper A K 2002 J. Chem. Phys. 1176196

[20] Benham M J, Cook J C, Li J-C, Ross D K, Hall P L and Sarkissian B 1989 Phys. Rev. B 39633

[21] Rønne C, Åstrand P-O and Keiding S R 1999 Phys. Rev. Lett. 822888

[22] Rønne C and Keiding S R 2002 J. Mol. Liq. 101199

[23] Bergman R, Swenson J, Børjesson L and Jacobsson P 2000 J. Chem. Phys. 113357

[24] Ryabov Y, Gutina A, Archipov V and Feldman Y 2001 J. Phys. Chem. B 1051845

[25] Gutina A, Antropova T, Rysiakiewicz-Pasek E, Virnik K and Feldman Y 2003 Microporous Mesoporous Mater. 58237

[26] D’Orazio F, Bhattacharja S, Halperin W P, Eguchi K and Mizusaki T 1990 Phys. Rev. B 429810

[27] Stapf S, Kimmich R and Seitter R-O 1995 Phys. Rev. Lett. 752855

[28] Korb J P, Malier J-P L, Cros F, Xu S and Jonas J 1996 Phys. Rev. Lett. 772312

[29] Holly R, Peemoeller H, Choi C and Pintar M M 1998 J. Chem. Phys. 1084183

[30] Shih P C, Lin H P and Mou C Y 2003 Stud. Surf. Sci. Catal. 146557

[31] Liu Y, Zhang W and Pinnavaia T J 2000 J. Am. Chem. Soc. 1228791

[32] Ryoo R, Joo S H and Kim J M 1999 J. Phys. Chem. B 1037435

[33] Copley J R D and Cook J C 2003 Chem. Phys. 292477 
[34] Meyer A, Dimeo R M, Gehring P M and Neumann D A 2003 Rev. Sci. Instrum. 742759

[35] Loong C-K et al 1987 Nucl. Instrum. Methods A 260381

[36] Fratini E, Chen S-H, Baglioni P and Bellissent-Funel M-C 2001 Phys. Rev. E 64 020201(R)

[37] Fratini E, Chen S-H, Baglioni P and Bellissent-Funel M-C 2002 J. Phys. Chem. B 106158

[38] Faraone A, Chen S-H, Fratini E, Baglioni P, Liu L and Brown C 2002 Phys. Rev. E 65040501

[39] Faraone A, Liu L, Mou C-Y, Shih P-C, Copley J R D and Chen S-H 2003 J. Chem. Phys. 1193963

[40] Liu L, Faraone A, Mou C-Y, Yen C-W and Chen S-H 2004 J. Phys.: Condens. Matter 16 S5403

[41] Chen S-H 1991 Quasi-elastic and inelastic neutron scattering and molecular dynamics of water at supercooled temperature Hydrogen Bonded Liquids ed J C Dore and J Teixeira (Dordrecht: Kluwer Academic) pp 289-332

[42] Bellissent-Funel M-C, Chen S-H and Zanotti J M 1995 Phys. Rev. E 514558

[43] Faraone A, Liu L, Mou C-Y, Yen C-W and Chen S-H 2004 J. Chem. Phys. 12110843

[44] Debenedetti P G and Stanley H E 2003 Phys. Today 56 (June) 40

[45] Anisimov M A, Sengers J V and Levelt Sengers J M H 2004 Near-critical behaviour of aqueous systems Aqueous Systems at Elevated Temperatures and Pressures: Physical Chemistry in Water, Steam and Hydrothermal Solutions ed D A Palmer, R Fernandez-Prini and A H Harvey (Amsterdam: Elsevier)

[46] Xu L, Buldyrev S and Stanley H E Private communication (The authors wish to acknowledge conversations with these researchers on Mar. 27, 2005 when they drew our attention to this interpretation of the $T_{\mathrm{L}}$ line)

[47] Kanno H, Speedy R J and Angell C A 1975 Science 189880

[48] Stanley H E 1999 Mysteries of water The Nato Science Series A vol 305, ed M-C Bellissent-Funel

[49] Angell C A, Borick S and Grabow M 1996 J. Non-Cryst. Solids 205-207 463

[50] Liu L, Chen S-H, Faraone A, Yen C-W and Mou C-Y 2005 Phys. Rev. Lett. 95117802

[51] Sastry S and Angell C A 2003 Nat. Mater. 2739

[52] Poole P H, Sciortino F, Essmann U and Stanley H E 1992 Nature 360324

[53] Ricci M A and Chen S-H 1986 Phys. Rev. A 341714

[54] Soper A K et al 2000 Phys. Rev. Lett. 842881

[55] Xu L, Kumar P, Buldyrev S V, Chen S-H, Poole P H, Sciortino F and Stanley H E 2005 Proc. Natl Acad. Sci. 10216558

[56] Maruyama S et al 2004 AIP Conf. Proc. 708675

[57] Brovchenko I, Geiger A and Oleinikova A 2005 J. Chem. Phys. 123044515 